\title{
Materials Evaluation Test Series (METS) 04, 05, and 06
}

David Zalk, Carl Ingram, Larry Simmons, Ron Arganbright, Jim Lyle, Keith Wong

March 30, 2006 
This document was prepared as an account of work sponsored by an agency of the United States Government. Neither the United States Government nor the University of California nor any of their employees, makes any warranty, express or implied, or assumes any legal liability or responsibility for the accuracy, completeness, or usefulness of any information, apparatus, product, or process disclosed, or represents that its use would not infringe privately owned rights. Reference herein to any specific commercial product, process, or service by trade name, trademark, manufacturer, or otherwise, does not necessarily constitute or imply its endorsement, recommendation, or favoring by the United States Government or the University of California. The views and opinions of authors expressed herein do not necessarily state or reflect those of the United States Government or the University of California, and shall not be used for advertising or product endorsement purposes.

This work was performed under the auspices of the U.S. Department of Energy by University of California, Lawrence Livermore National Laboratory under Contract W-7405-Eng-48. 


\title{
Material Evaluation Test Series (METS) 04, 05, and 06
}

\author{
PIs: D. Zalk', C. Ingram², L. Simmons' ${ }^{2}$, \\ Co-PIs: R. Arganbright ${ }^{3}$, J. Lyle ${ }^{2}, K$. Wong ${ }^{2}$ \\ ${ }^{1}$ HCD, SEP \\ ${ }^{2 \cdot}$ Bdiv, DNT \\ ${ }^{3}$ MMED
}

\begin{abstract}
The purpose of this work is to examine the environmental, safety, health and operational aspects of detonating a confined explosive test apparatus that has been designed to maximize the dynamics of impact on beryllium metal components for Contained Firing Facility (CFF) applications. A combination of experimental collection and evaluation methods were designed and implemented to provide an evaluation of immediately postdetonation by-products reflecting a potential worst-case scenario beryllium aerosolization explosive event. The collective Material Evaluation Test Series (METS) 04-06 provided explosive devices designed to scale for the dedicated METS firing tank that would provide a post-detonation internal environment comparable to the CFF. The experimental results provided appropriate information to develop operational parameters to be considered for conducting full-scale beryllium-containing experimental tests with similar designs within $\mathrm{CFF}$ and B801A. These operational procedures include the inclusion of chelating agents in pre-shot CFF cardboard containers with a minimum of 600 gallons content, an extended time period post-test before purging the CFF chamber, and an adaptation of approaches toward applications of the scrubber and HEPA systems during the post-shot sequence for an integrated environmental, safety, and health approach. In addition, re-entry and film retrieval procedures will be adapted, in line with abatement techniques for cleaning the chamber, that will be required for work inside a CFF that will contain an elevated concentration of spherical and highly aerosolizable beryllium particulate.
\end{abstract}

\section{INTRODUCTION}

\section{History}

Since the early 1970's several national laboratories have spent tens of millions of dollars attempting to learn what happens to beryllium during and after an explosive event. The determination of beryllium aerosolization as a result of dynamic experiments has therefore been a research objective over the last four decades. Investigations into the aerosolization of beryllium begin with the LASL dynamic experiments (Dahl and Johnson 1977). This research then continues at LLNL with outdoor beryllium dispersion evaluations near explosive firing tables (Shinn 1989) and the measurement and dispersion of beryllium from high explosive tests at Site 300 (Baskett 1991). Additional research into beryllium aerosolization from outdoor dynamic tests has also been performed internationally in the 1990's (Citation restricted). It has been quite difficult to apply this 
information to the needs of Site 300 and CFF as the calculations performed in determining even generalized beryllium aerosolization potential are scant, collection of aerosolized materials in open-air experiments has been proven historically to be difficult to achieve, and agreement and interpretation of results among the laboratories is rare. For the needs of the Material Evaluation Test Series (METS) research team, the vast majority of this collective historical information is considered to be inapplicable. This inapplicability is due to ineffective capture and analysis methods that have resulted in difficulty finding metallic beryllium or its compounds released during open-air dynamic testing and have led to resultant conclusions of either a lack of aerosilizable beryllium or gross estimates of fractionated beryllium expressed as a percentage $(1-10 \%)$ of its predetonation mass within a broad range of dynamic test designs and therefore no accurate conclusions are recorded.

\section{METS Research Team}

With this dearth of information relating to characterization of toxic materials aerosolized and released to the environment from explosives testing a multidisciplinary, interdepartmental research team positioned themselves to conduct this research to determine these parameters to assist in the operation of the CFF within B801A. This system was first put into application for the collection of metals during the METS $01-03$ conducted in 2001 - 02 (Zalk 2002) for scaled explosive devices designed to transfer a moderate shock to beryllium-containing dynamic test devices in METS 02 - 03. Our primary purpose for these experiments was to acquire as much knowledge as possible about beryllium surface and air contamination levels in an internal post-explosive environment to assess potential for personal exposures, area air, and surface area dispersal to develop and implement rapid decontamination of firing chambers. These experimental metals analysis techniques were developed during the CFF Structural Qualification Test Series (SQTS) utilizing aluminum as a surrogate for beryllium (Zalk 2001). Subsequent to these early METS activities we have successfully performed feasibility studies on full-scale dynamic events within CFF to perfect techniques associated with immediate post-detonation materials capture, analysis, morphology, particle sizing, speciation, and dispersible characteristics of fractionated and vaporized hazardous metals (Zalk et al 2003). Toward this end we had designed a system that could collect, filter, separate, and analyze aerosolized materials from measured volumes of a scaled firing tank's contents after dynamic events relating to highly hazardous metals during METS 07 - 09 (Zalk et al 2004). This collection system was further perfected and adapted to best capture beryllium at measured and maintained concentrations within a fraction of a minute immediately post-detonation to achieve the parameters necessary for the proposal requirements of METS $04-06$.

\section{SCOPE AND OBJECTIVES}

This study is focused on developing an appropriate characterization of immediate postdetonation and post-experiment beryllium aerosolization presented by worst-case scenario explosive dynamics on beryllium components within an internal environment. The detonations occur within a 314 cubic foot cylindrical firing tank. The tank is rated 
for 500 grams of energetic material. To accomplish the measurement and evaluation of beryllium particulate aerosolization potential, collection and analysis techniques were developed to capture particulates generated within the first 15 and 30 seconds immediately post-detonation, a window of time that begins to approach explosive dynamics analogous to an outdoor dynamic event. Utilizing the same experimental equipment, the subsequent $21 / 2$ to 5 minute intervals of the post-detonation environment are captured, a window of time that is most comparable to the window that leakage has been historically seen to occur from within CFF into the B801A Accidental Detonation Hazard Zone (ADHZ) and the common areas of this facility. This METS $04-06$ experimental focus on beryllium is multi-factorial, however there is a primary concern for the occupational health dynamics of preventing Chronic Beryllium Disease (CBD) and its systematic relation to the B801 complex and its employees.

CBD is a progressive lung disease with granulomas and fibrosis as part of its characterization, occurring in individual who are sensitized to beryllium (Maier and Newman 1998). Insoluble beryllium exposure has been tied to CBD (Kriess et al 1997, Eisenbud 1998), however a direct and consistent exposure-response relationship to CBD has been elusive using current total dust aerosol personal measurement techniques. Current research indicates the potential for properties relating to particle size and surface area to be more appropriate designators for determining the bioavailability of beryllium toward the risk of acquiring CBD (Stefaniak et al 2004). Further, currently unproven hypotheses have been published that point toward an immunological route of initial beryllium sensitization linked to dermal exposure of insoluble beryllium and the direct skin penetration of particulate smaller than $1 \mu \mathrm{m}$ (Tinkle et al 2003).

\section{Research Goals}

The METS $04-06$ proposal sought to address these requirements and detection parameters to best mitigate this component for the B801 complex and its environmental and personnel exposure concerns. A research protocol for the detection, collection, and characterization of aerosolized beryllium particulates, both immediately post-detonation and during worker clean-up procedures, was developed to assist in the determination of the appropriate response for full-scale dynamic test parameters to control the experimental by-products, predict fugitive emissions and chamber purging constituency. In addition, practically it is necessary to learn how to preventatively control beryllium personal exposure potential while cleaning the CFF with properly designated personal protection equipment to prepare the chamber for timely re-use. The following research specific goals were presented (Zalk et al 2005):

1. Understanding worst-case scenario constituency of beryllium relating to leak potential within B801A ADHZ and common areas.

2. Determining the particle size distribution of worst-case scenario berylliumcontaining dynamic experiments.

3. Determine and separate beryllium metal and beryllium oxide content and size distribution both immediately post-detonation and during clean-up procedures. 
4. Predicting beryllium morphology (spherical vs. non-spherical) to answer conflicting expert advice on whether we are working with fractionalization or vaporization mechanics as a part of the newly created post-detonation science.

5. Utilize collective analytical information to determine if a similar full-scale dynamic experiment within the CFF can be performed successfully.

6. Outline the protocol and procedures, based on experimentally-obtained information, to control airborne and surface migration of beryllium particulate and/or vapor contamination immediately post-detonation, during re-entry, film retrieval, and chamber clean-up activities, and as a part of ongoing facility maintenance and operations over time.

\section{Research Parameters}

In order to achieve these specific research needs, a series of research parameters required development. These include the development, perfecting, and implementation of postdetonation capture equipment that would yield replicate samples under the variety of conditions relating to dynamic testing. Subsequent to successful capture of metal analysis samples, an appropriate array of investigative and scientific methods would need to be combined to overcome limitations relating to beryllium detection and speciation with available analytical methods and tools. Building on the previous developments and successes, discussed above, by the METS research team, the following research parameters were outlined (Zalk et al 2005):

1. Perform METS $04-06$ experiments within existing firing tank, on the B812 firing table, using newly modified and updated remote sampling equipment.

2. Evaluate beryllium experimental parameters based on worst-case scenario dynamic test component locations under appropriately scaled test conditions.

3. Evaluate beryllium characteristics based on low to high-pressure parameters.

4. Develop and perfect new sampling, collection and analytical methods to validate our research needs and field application requirements.

5. Create, develop, and perfect the research needs and parameters for future indoor chamber sampling protocol to achieve results analogous to outdoor dynamic events.

6. Create, develop and perfect analytical information to provide important information for authorization requirements for outdoor experiments.

7. Offer the collection and evaluation mechanism to afford the ability to test different metals and gasses associated with individual shot design parameters.

\section{EXPERIMENTAL METHODS}

\section{Post-Detonation Collection System}

The detonations occur within a 314 cubic foot steel cylindrical firing tank capable of igniting up to 500 grams of energetic material. This firing tank was used to determine the 
METS testing for beryllium metal on the B850 firing table. This tank was transferred to the B812 firing table to enable this extended experimental process (Figure 1).

Figure 1. Firing tank configuration for METS 04A - 06A on the B812 firing table.

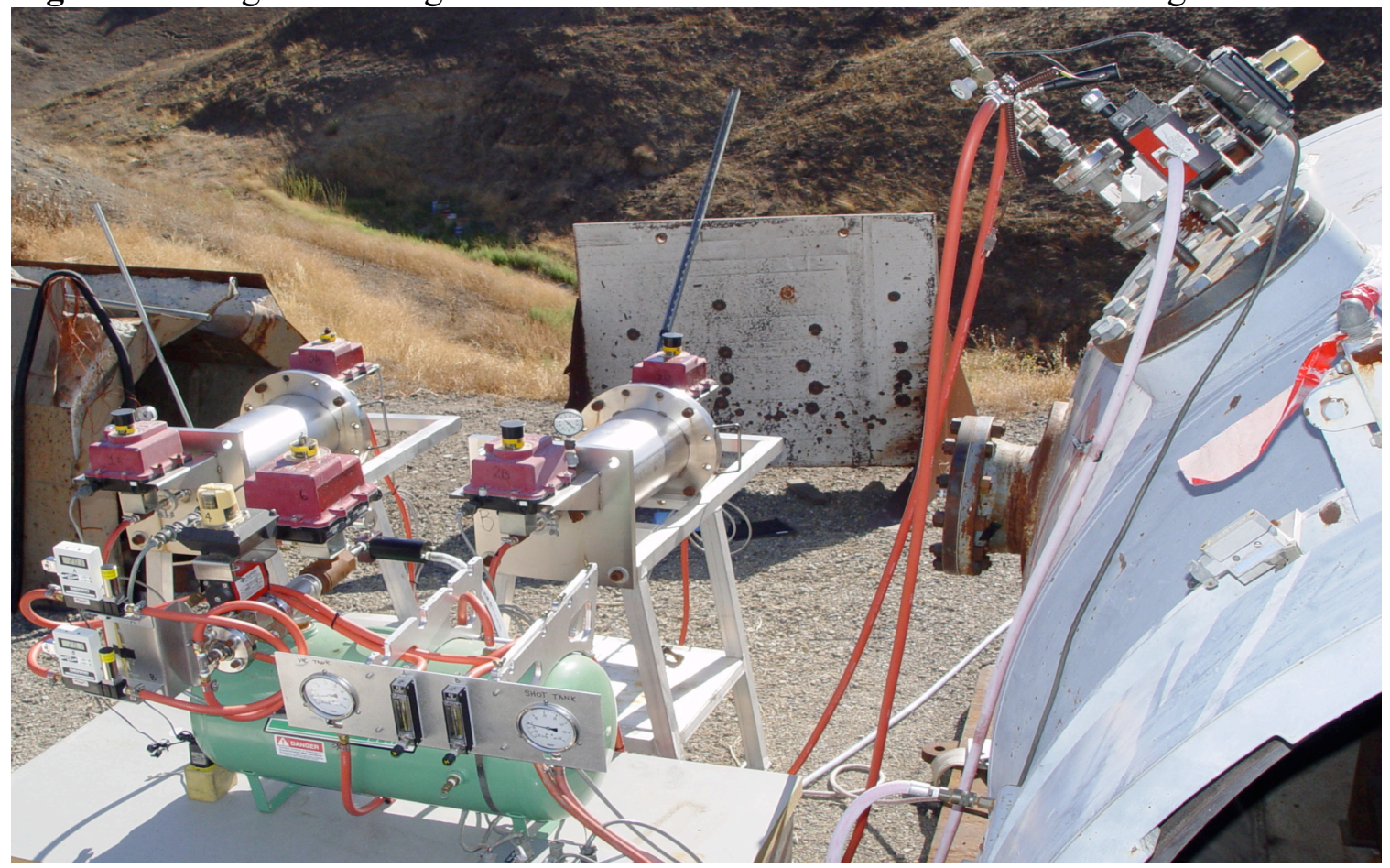

The system is designed to collect and filter a measured quantity of chamber atmosphere after a dynamic event in order to characterize aerosolized materials that may be trapped on the filters. Four collecting vessels are used so that changes in particulate concentration can be determined as a function of time after an event. The flow of the gas into each collecting vessel is divided into four filtered streams. The filters are easily removed and sent to analytical laboratories for analysis. No single analysis technique will suffice for all the materials of interest or their compounds, but four nearly identical filters are sufficient for our purposes.

\section{Plumbing:}

Gas is drawn through a 1-inch pipe that penetrates the wall of the firing chamber into a manifold that is fitted with four collection cassettes. Various remote-controlled valves are used to direct the flow into and out of the two vessels (Tank A and Tank B). Filtered gas flows out of the vessels through an analog flow meter and then through a digital flow controller, then subsequently into ambient air.

Measurements, signal sources:

Due to the flow meter and controller, the previous need to measure gas temperature and pressure verses time, at several locations in the system, was not necessary. The time that gas flows in a vessel can be selected manually. The valves to all two or a lesser number of vessels can be ganged, i.e. operated at the same time. The entire time of data collection can be as long as 1hour. 
- $\quad$ Firing chamber pressure vs. time to vent (existing).

- $\quad$ Firing chamber temperature vs. time to vent (existing)

- $\quad$ Two thermocouples. Expected temperature ambient to $1000{ }^{\circ} \mathrm{F}$.

- $\quad$ Three pressure transducers. Expected pressure 0 psia to 50 psia

- $\quad$ Flow rate and total flow. The mass flow controller is calibrated to vacuum, and not to ambient air, and it is placed in series with a flow meter. Potential error to this system is discussed further below.

Remotely Controlled Valves:

- 8 valves in the filter assy. Type TBD, likely $24 \mathrm{v}$ AC, or $110 \mathrm{v}$ AC solenoid

- 2 globe valves, one at the exit of the pipe into the chamber, the other at the vacuum pump.

Programmable Requirements:

- Valve opening and closing times after an event, 1 second to 1 hour.

- Manual switch bypasses to over-ride the valve program.

Console Indicators gauges, bypass switches:

- $\quad$ Valve number and condition, open/closed, (panel light green/red).

- $\quad 2$ channels temperature, ambient to $1000^{\circ} \mathrm{F}$

- 3 channels pressure, 0 to 50 psia

- 12 bypass switches (2 spares)

\section{Firing Tank Collection Apparatus}

Three devices were built to evaluate material exposed to worst-case scenario, explosively driven shock and thermal effects to beryllium components. The design for METS 04A and 05A had approximately 15 times more initial beryllium mass than METS 06A (More detailed information can be found in the classified version of this document). Each of the beryllium-containing devices (METS 04A, 05A, and 06A respectively) were placed within the B812 firing tank (example in Figure 2) and detonated August through September 2005 and METS 06C calibration test in November 2005. Each of these METS dynamic events were captured immediately post-detonation, at varying time intervals, and captured on two sets of four unique $41 \mathrm{~mm}$ Whatman $0.8 \mu \mathrm{m}$ mixed cellulose ester filters that separated particulate from the airstream to create replicate samples provided by a four-branch separator (Figure 3). This initial experimental collection system (Figures 4 and 5) was developed to narrow the capture window immediately post-detonation to evaluate the beryllium characteristics relating directly to dynamic event parameters within the chamber. The flow rate for the capture system was continuously maintained at $20 \mathrm{l} / \mathrm{min}$ using both analog and digital methods. The first four-branch separator served to collect the 30-second (METS 04A) and 15 second (METS 05A and 06A) window immediately post-detonation to begin research into analogous sampling parameters for outdoor dynamic events. 
Figure 2. Example of a METS-related device set-up within the firing tank.

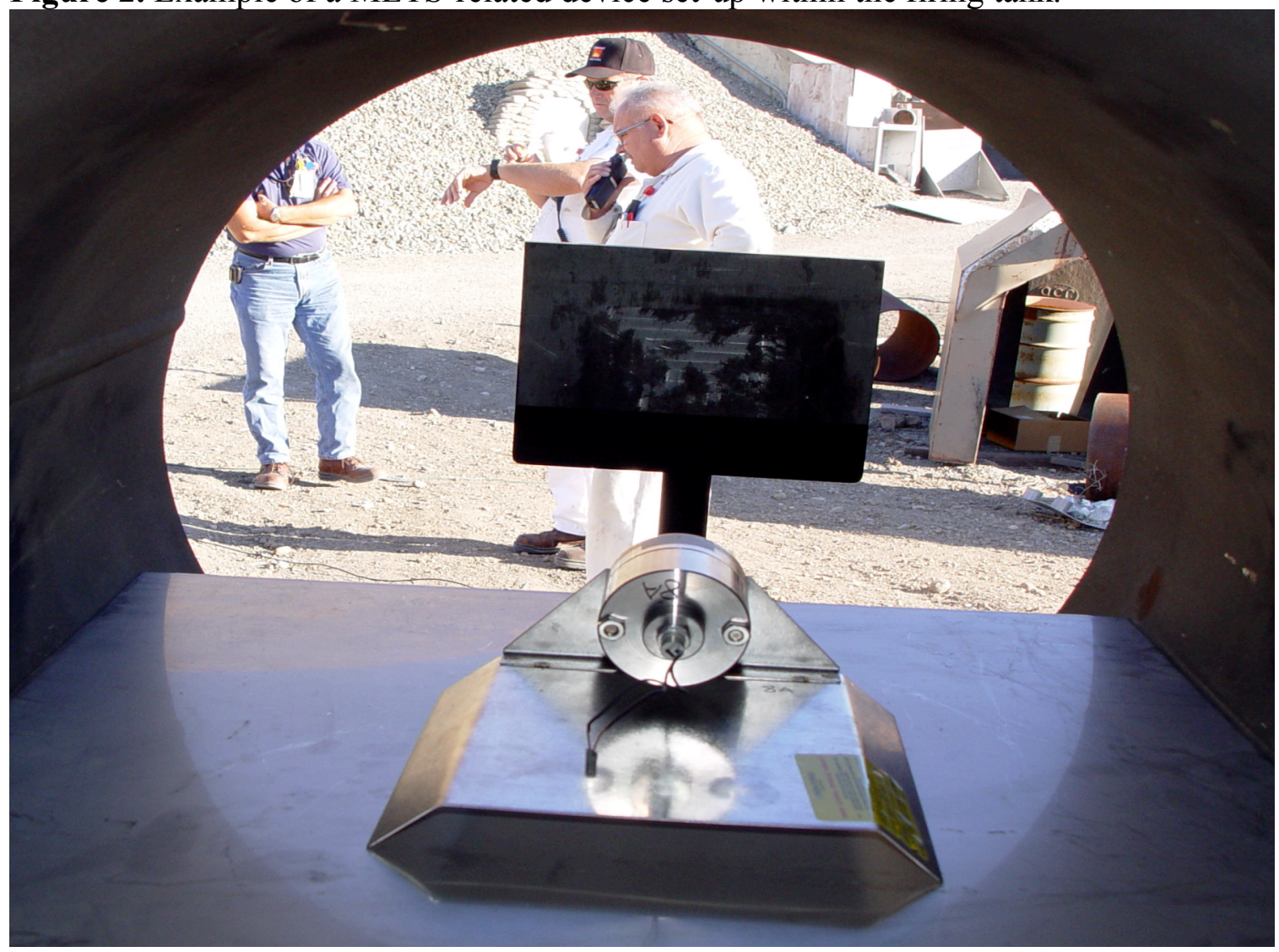

Figure 3. METS 04A - 06A four-branch sample collection and replication apparatus.

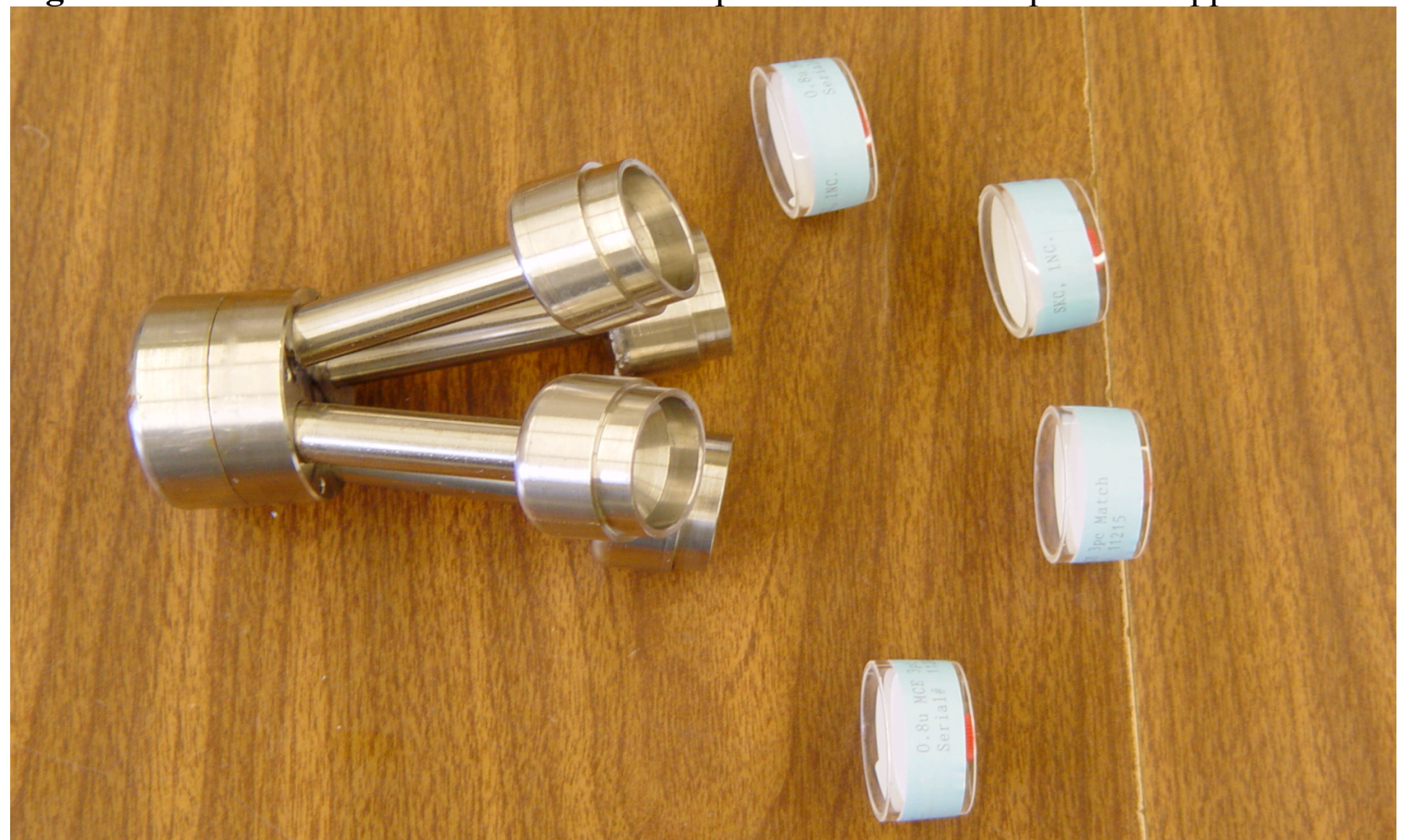


Figure 4. Schematic of the METS 04A - 06A collection system apparatus.

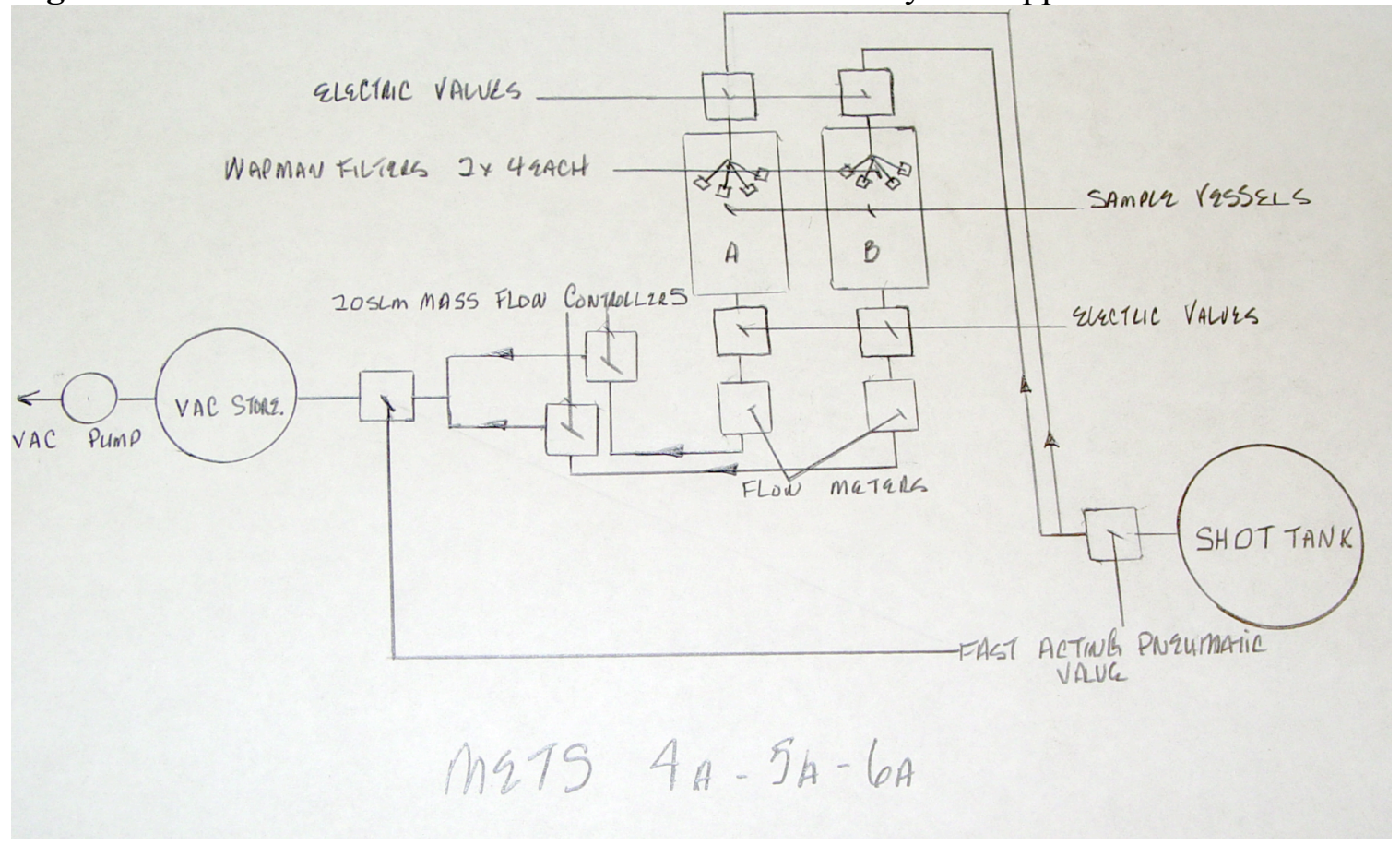

Figure 5. Photograph of METS 04A - 06A collection system apparatus.

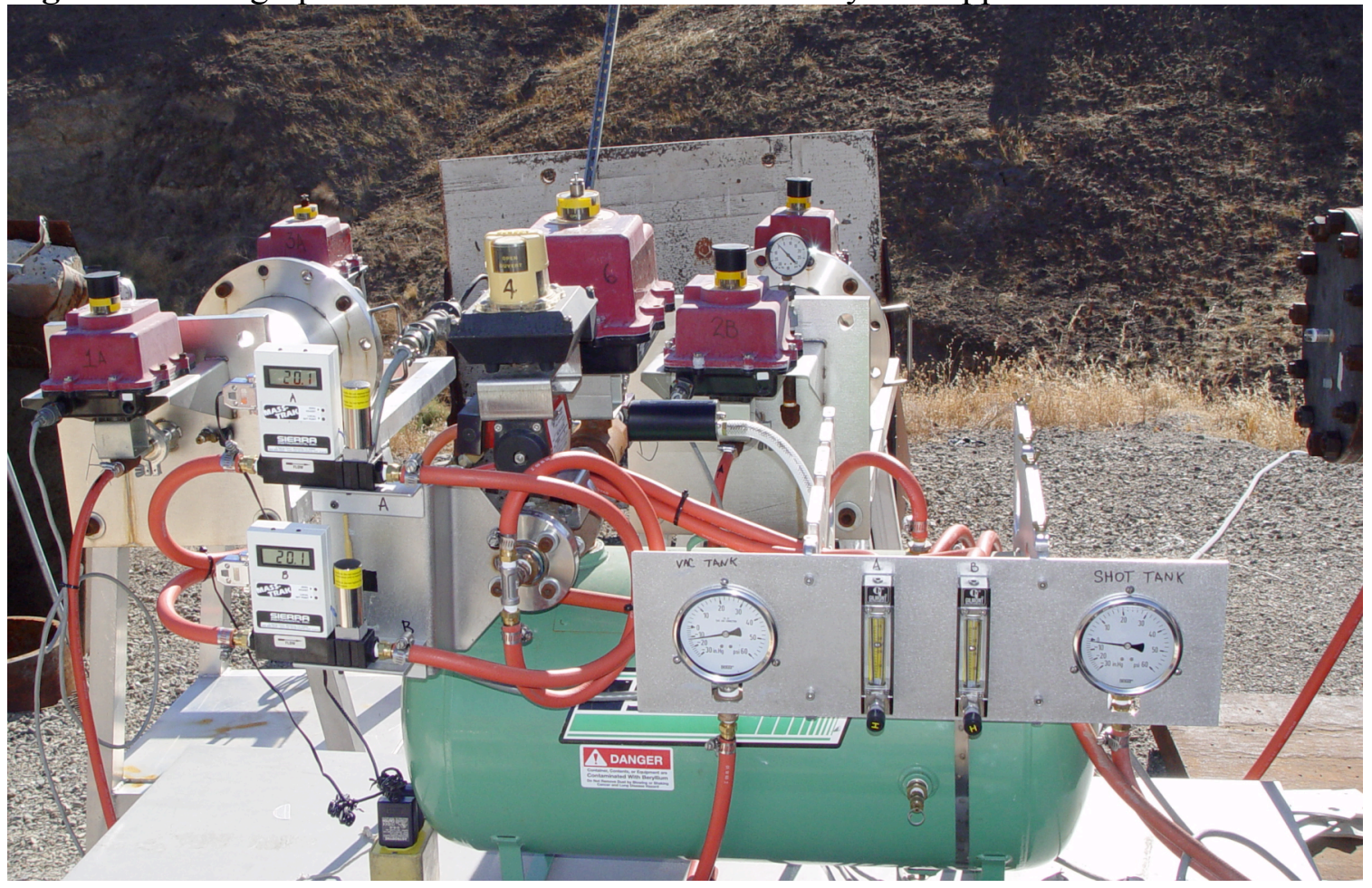


The second four-branch separator served to collect the subsequent four and one half minutes (METS 04A) and two and one half minutes (METS 05A and 06A) to serve as an airstream constituency most applicable to CFF fugitive emissions relating to chamber leakage in B801A historically. For each of the four-branch separator sampling cassettes, one will be analyzed for total beryllium (beryllium metal and its oxide) and a replicate will be analyzed for beryllium metal specifically.

\section{Chamber Environment}

After a minimum of 24 hours post-detonation the firing tank door is opened for the first time after the particulate has been given the same opportunity to settle as prescribed within the CFF chamber procedures. Placed inside the firing tank at this time are duplicate cascade impactors with additional personal air sampling devices. These sampling devices include traditional total dust collection methods as well as experimental evaluation of various inhalable and respirable size-selective collection devices available for a couple of experimental testing opportunities during METS 05 and 06. All samples were taken simultaneously in a homogenously mixed internal tank environment. Mixing utilized a hollow metal tube system, with 10 equally spaced holes along its bottom, to remotely distribute 100 psi hydraulically driven air across an approximate 3 foot by 1 foot area of the closed firing tank floor's surface for a specified period of time. The mixing system and the cascade impactors ran for 5 minutes during METS 04A and both ran for 20 minutes during METS 05A and 06A. The cascade impactors and the mixing systems were then turned off while the remaining personal sampling devices continued to run for a minimum of an additional 30 minutes as the homogeneously mixed environment was afforded time to settle. After settling, the firing tank door is opened again and personal sampling devices were turned off and removed.

Once the ambient chamber environment has been sampled, the procedures for the lowpressure washing, HEPA vacuuming, and initial cleaning of the internal firing tank are performed by established firing table personnel. These employees wear appropriate protective gear and personal sampling pumps to reflect the potential for exposure for those performing similar work. Although these workers remain outside the firing tank at all times and do not enter the firing tank prior to its full cleaning, the personal exposure levels are expected to be a rough estimate due to the confined nature of the surfaces cleaned. After each of the METS 04A - 06A beryllium-containing devices' contamination of the internal firing tank had been thoroughly washed cleaned, a second test-associated $\mathrm{C} 4$ explosive device was placed within the firing tank. These additional explosive devices, METS 04B, 05B, and 06B, were then detonated to serve as a flush of the internal firing tank's surfaces to remove a significant portion of the background surface beryllium levels that may have remained after the completion of the first cleaning procedures. The contamination relating to these explosive flushes of the firing tank is then cleaned in a similar manner as used subsequent to the initial explosive events to reduce potential surface contamination of beryllium from one METS test to the next to near background levels. 
Collection System Calibration (METS 06C)

After the completion of the METS 06B tank flush and its cleaning, an additional METS $06 \mathrm{C}$ simulated detonation was performed within the firing tank to serve as a calibration of this research's collection systems. This calibration was performed with aluminum powder utilizing the mass and size fraction of aluminum powder best corresponding to the theory that the entire mass of beryllium in the METS 04A device had been vaporized. Equal mass portions of $<1$ micron, $4.5-7$ micron, and $10-14$ micron aluminum powder were tare weighted and added to a $100 \mathrm{ml}$ glass beaker to equal to the mass of beryllium in the METS 04A device, which by design and mass was most likely to create the highest concentration of aerosolizable beryllium vapor and/or particulate of this METS experiment. The "detonation" of this aluminum surrogate was performed with a pressurized air source, initiated with a fast acting valve, that rapidly evacuating the aluminum powder at an equivalent three-foot level above the firing tank's floor. This "detonation" simulation was verified external to the firing tank using cornstarch and was verified by a firing table expert to be visually appropriate. Immediately post"detonation", the same collection device sequence was performed as in METS 05A and $06 \mathrm{~A}$ with the both of the four-branch samplers and subsequently the cascade impactors and personal sampling devices. The theory put forward prior to this calibration test was that if complete vaporization of the beryllium in METS 04A occurred, we should be able to have a similar recovery ratio of aluminum both immediately post-detonation and in a homogeneously mixed firing tank environment.

\section{Sampling Equipment and Analytical Methods}

\section{Post-Detonation Collection Tanks}

Both collection tanks are set up so that parallel flow rates of 20 liters per minute $(1 / \mathrm{min})$ are obtained. There are two collection vessels (Tanks A\&B) that each have a four-branch airstream separator with four sample collection cassettes, each receiving $5 \mathrm{l} / \mathrm{min}$ for replicating a given airstream. Each of the four-branch airstream separator stems is a sample cassette equipped with a 41 millimeter, 0.8 -micron pore size, mixed cellulose ester fiber (MCEF) filter. Sample analysis for total beryllium was performed by Inductively Coupled Plasma - Atomic Emission Spectrometry (ICP-AES) utilizing an InHouse ORNL Method to ensure the analysis includes both beryllium metal and beryllium oxide; Metals such as beryllium and aluminum were included in the Industrial Hygiene metal series analyzed according to the NIOSH Method 7300. The analyses were performed by the LLNL Hazards Control Analytical Laboratory, an American Industrial Hygiene Association (AIHA) accredited laboratory. The analytical results listed in Table 1 are results received. However, due to the nature of the collection system's connection to vacuum tanks and not to an ambient air environment, a sampling system error has been derived so it can be applied throughout this table. The initial mass flow controller is listed for an accuracy reading of $1.5 \%$ with an outlet pressure certified to 23 inches mercury gauge (" $\mathrm{Hg} \mathrm{g}$ ). This flow controller, factory calibrated for accuracy at $+/-5 \%$, is placed in series with a rotameter calibrated against a primary standard. Therefore, it is a conservative assumption that the system should have a total of $+/-10 \%$ throughout. 
This error should be uniform throughout due to consistency in application of the system's design parameters.

\section{Cascade Impactor}

Thermo Anderson 20-830 non-viable eight stage respirable range (0.4 to 9.0 micron) impactor in standard configuration were placed on the firing table floor adjacent to the door, and were connected in-line with area air sampling pumps performing at $28.3 \mathrm{l} / \mathrm{min}$ utilizing a precision rotameter previously calibrated against a primary standard. Each of the stages contained equipment sized 0.8-micron pore size, MCEF filters and were connected inline with Tygon ${ }^{\circledR}$ tubing. A post-survey flow rate was performed to check for change in sampling rate due to build-up on the cassette filter. Sample analysis for total beryllium was performed by Inductively Coupled Plasma - Atomic Emission Spectrometry (ICP-AES) utilizing an In-House ORNL Method to ensure the analysis includes both beryllium metal and beryllium oxide; Metals such as beryllium and aluminum were included in the Industrial Hygiene metal series analyzed according to the NIOSH Method 7300. The analyses were performed by the LLNL Hazards Control Analytical Laboratory, an American Industrial Hygiene Association (AIHA) accredited laboratory.

Personal and Surrogate Air Monitoring Gilian personal air sampling pumps were placed for METS 04A, 05A, 06A, and 06C at a height of 5 feet above the tank floor to replicate personal air monitoring at the breathing zone performed at a rate of $3.0 \mathrm{l} / \mathrm{min}$ utilizing a precision rotameter previously calibrated against a primary standard. The same pumps, flow rates, and protocol are also used for the personal sampling opportunities as part of this experiment's procedures. CIP-10 inhalable foam samplers were placed on the firing tank floor, next to the cascade impactors, during METS 05A and 06A and were calibrated per manufacturer specifications which are not flow rate dependent in a manner similar to traditional in-line air sampling. GSA-GSP inhalable samplers equipped with 37 millimeter, 0.8-micron pore size, MCEF filters were suspended at 5 feet above the tank floor during as replicate samples during METS 06A and performed at rates of $3.51 / \mathrm{min}$ utilizing a precision rotameter previously calibrated against a primary standard. IOM samplers, which contain both an inhalable foam core center and a respirable fraction MCEF 0.8-micron pore size collection filter in the same unit, were suspended 5 feet above the tank floor during METS 05A and 06A and performed at a rate of $2.0 \mathrm{l} / \mathrm{min}$ utilizing a precision rotameter previously calibrated against a primary standard. Cassettes for personal air and analogous internal tank area air sampling were 37-millimeter, 0.8-micron pore size, mixed cellulose ester fiber $(\mathrm{MCEF})$ filter and were connected inline with Tygon ${ }^{\circledR}$ tubing. A post-survey flow rate was performed to check for change in sampling rate due to build-up on the cassette filter. Sample analysis for the Gilian personal sampling pumps total dust cassettes was both total beryllium, performed by Inductively Coupled Plasma - Atomic Emission Spectrometry (ICP-AES) utilizing an In-House ORNL Method to ensure the analysis includes both beryllium metal and beryllium oxide; and the IH metal series (personal simulation cassettes only) was analyzed according to the NIOSH Method 7300. These analyses were performed by the LLNL Hazards Control Analytical Laboratory, an AIHA accredited laboratory. The analysis of the other inhalable and respirable personal 
sampling devices was performed according to the NIOSH Method 7300 for beryllium metal by DataChem Laboratories of Salt Lake City, Utah, also an AIHA accredited laboratory.

\section{Surface Swipe Sampling}

Surface swipe samples were performed wet, utilizing dionized water on Whatman 41 paper tabs. Sample analysis for total beryllium was performed by Inductively Coupled Plasma - Atomic Emission Spectrometry (ICP-AES) utilizing an In-House ORNL Method to ensure the analysis includes both beryllium metal and beryllium oxide. The analyses were performed by the LLNL Hazards Control Analytical Laboratory, an American Industrial Hygiene Association (AIHA) accredited laboratory.

\section{Scanning Electron Microscopy}

The Scanning Electron Microscopy (SEM) was performed in house at LLNL on less than one square centimeter fully encapsulated samples embedded on MCEF utilizing a Hitachi $\mathrm{S}-4500$, cold field emission scanning electron microscope. All images provided were shot with a secondary electron detector. EDAX energy dispersive X-ray microanalysis system with an ultra-thin beryllium window was used for qualitative chemical analysis, with an X-ray source than cannot identify beryllium particulate specifically. The standard-less quantitative analysis for the metals presented below was performed with EDAX Genesis Spectrum Quant ZAF, version 1.0.

\section{RESULTS}

\section{Post-Detonation Collection Tanks}

Each of the beryllium-containing devices (METS 04A, 05A, and 06A respectively) were placed within the B812 firing tank and detonated August through September 2005. Each of these METS dynamic events were captured immediately post-detonation, at varying time intervals, based on the firing tank collection system described above. Tank A (primary) and Tank B (secondary) each captured one set of four unique $41 \mathrm{~mm}$ Whatman $0.8 \mu \mathrm{m}$ mixed cellulose ester filters that separated particulate from the airstream to create replicate samples that were analyzed for both total beryllium (metal and oxide) and beryllium metal. This initial experimental collection system was developed to narrow the capture window immediately post-detonation to evaluate the beryllium characteristics relating directly to dynamic event parameters within the chamber. The first four-branch separator served to collect the 30-second (METS 04A) and 15-second (METS 05A and 06A) window immediately post-detonation to begin research into analogous sampling parameters for outdoor dynamic events. Analytical results for digested MCEF filters obtained from these capturing systems are presented individually in Table 1 and are presented as comparisons in Figure 6. SEM visual imaging results for the identified sample sequence are presented in Figures $7 \mathrm{~A} \& \mathrm{~B}-10 \mathrm{~A} \& \mathrm{~B}$. 
Table 1; Collection levels immediately post detonation for beryllium metal and total beryllium (metal and oxide) for these METS experiments. Compare to Action Limit of $0.2 \mu \mathrm{g} / \mathrm{m}^{3}$ as Total Beryllium.

\begin{tabular}{|c|c|c|}
\hline $\begin{array}{c}\text { Sample Date } \\
\text { Sample Sequence } \\
\text { Batch \# } \\
\text { Sample \# }\end{array}$ & $\begin{array}{l}\text { Total Beryllium } \\
\mathrm{mg} / \mathrm{m}^{3 *}\end{array}$ & $\begin{array}{l}\text { Beryllium Metal } \\
\mathrm{mg} / \mathrm{m}^{3}\end{array}$ \\
\hline $\begin{array}{c}\text { 8/17/05 } \\
\text { METS 04A Capture Tank A } \\
\text { Batch\# 20080173, } 20080176 \\
\text { FS\#2111524, 2111529 }\end{array}$ & $\begin{array}{c}78.4^{* *} \\
(30 \text { seconds post- } \\
\text { detonation })\end{array}$ & $\begin{array}{c}79.2 \\
\text { (30 seconds post- } \\
\text { detonation) }\end{array}$ \\
\hline $\begin{array}{c}8 / 17 / 05 \\
\text { METS 04A Capture Tank B } \\
\text { Batch\# 20080173, } 20080176 \\
\text { FS\#2111525, 2111530 }\end{array}$ & $\begin{array}{c}38.1 \\
\begin{array}{c}\text { (Subsequent } 5 \text { minutes } \\
\text { post-detonation) }\end{array} \\
\end{array}$ & $\begin{array}{c}41.6 \\
\begin{array}{c}\text { (Subsequent } 5 \text { minutes } \\
\text { post-detonation) }\end{array} \\
\end{array}$ \\
\hline $\begin{array}{c}\text { 8/25/05 } \\
\text { METS 05A Capture Tank A } \\
\text { Batch\# 20080344, 20080346 } \\
\text { FS\#2111628, 2111631 }\end{array}$ & $\begin{array}{c}57.6 \\
\text { (15 seconds post- } \\
\text { detonation) }\end{array}$ & $\begin{array}{c}54.4 \\
\text { (15 seconds post- } \\
\text { detonation) }\end{array}$ \\
\hline $\begin{array}{c}\text { 8/25/05 } \\
\text { METS 05A Capture Tank B } \\
\text { Batch\# 20080344, 20080346 } \\
\text { FS\#2111629, } 2111632\end{array}$ & $\begin{array}{c}14.4 \\
\text { (Subsequent 2 1/2 } \\
\text { minutes post-detonation) }\end{array}$ & $\begin{array}{c}14.4 \\
\text { (Subsequent 2 } 1 / 2 \\
\text { minutes post-detonation) }\end{array}$ \\
\hline $\begin{array}{c}9 / 01 / 05 \\
\text { METS 06A Capture Tank A } \\
\text { Batch\# 20081740 } \\
\text { FS\#2112015 }\end{array}$ & $\begin{array}{c}11.2 \\
\text { (15 seconds post- } \\
\text { detonation) }\end{array}$ & N/A \\
\hline $\begin{array}{c}\text { 9/01/05 } \\
\text { METS 06A Capture Tank B } \\
\text { Batch\# } 20081740 \\
\text { FS\#2112016 }\end{array}$ & $\begin{array}{c}2.7 \\
\text { (Subsequent 2 1/2 } \\
\text { minutes post-detonation) }\end{array}$ & N/A \\
\hline
\end{tabular}

$* \mathrm{mg} / \mathrm{m}^{3}$; milligrams per cubic meter of air

** These analytical sample results are presented as received. However, due to the nature of the collection system, it is feasible to account for a $+/-10 \%$ sampling system error throughout this table. This error should be uniform throughout due to consistency in application of the system's design parameters. 
Figure 6; Collection comparisons between the METS 04A - 06A beryllium-containing tests (Table 1) are presented. Collection levels immediately post detonation* for beryllium metal and total beryllium (metal and oxide) for these METS experiments. Compare to Action Limit of $0.2 \mu \mathrm{g} / \mathrm{m}^{3}$ as Total Beryllium.

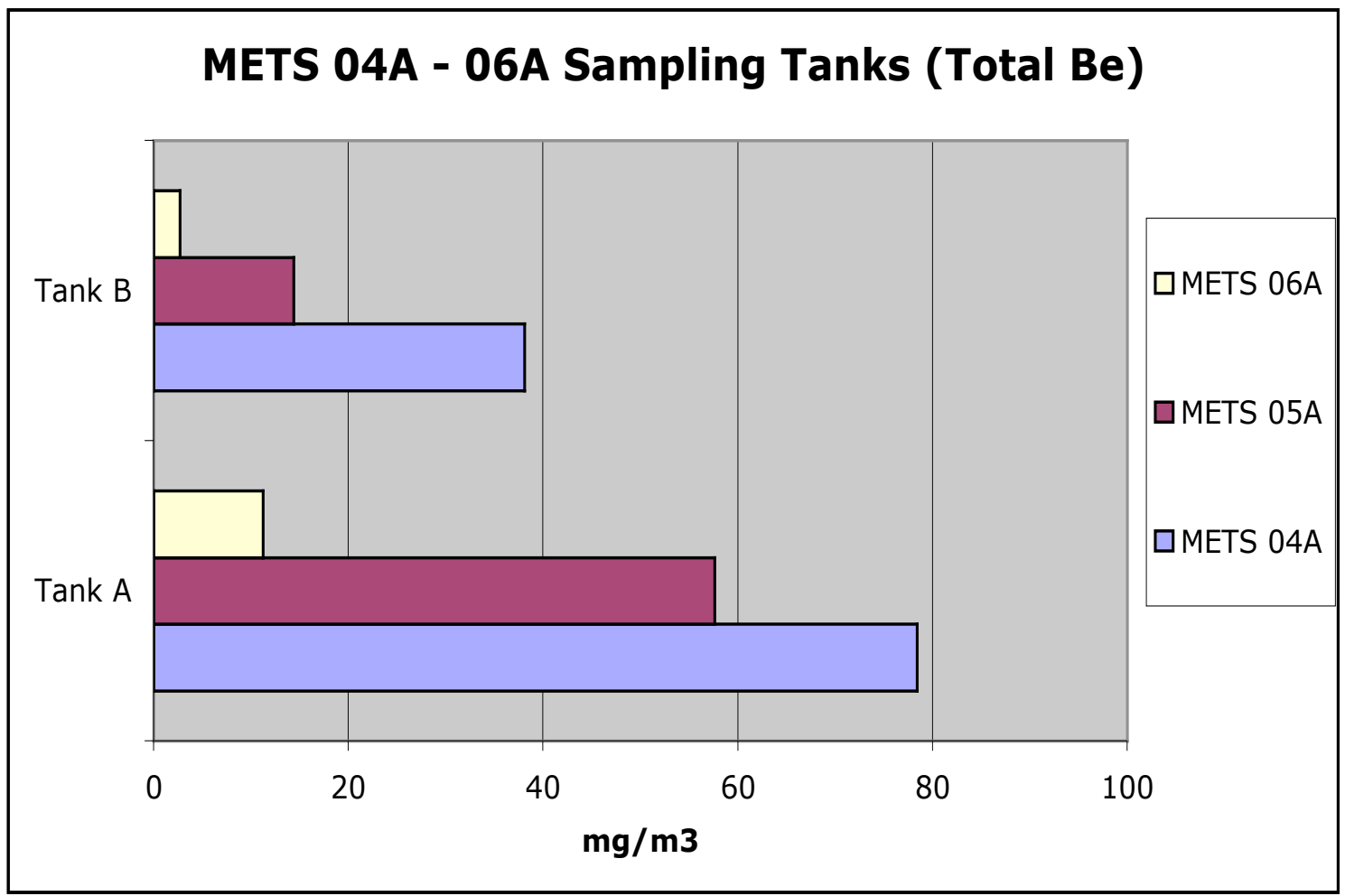

* These analytical sample results (derived from Table 1) are presented as received. However, due to the nature of the collection system, it is feasible to account for a $+/-10 \%$ sampling system error throughout this table. This error should be uniform throughout due to consistency in application of the system's design parameters. 
Figures 7A\&B; METS 04A Tank A; Collected 30 seconds post-detonation at $\sim 20$ microns.

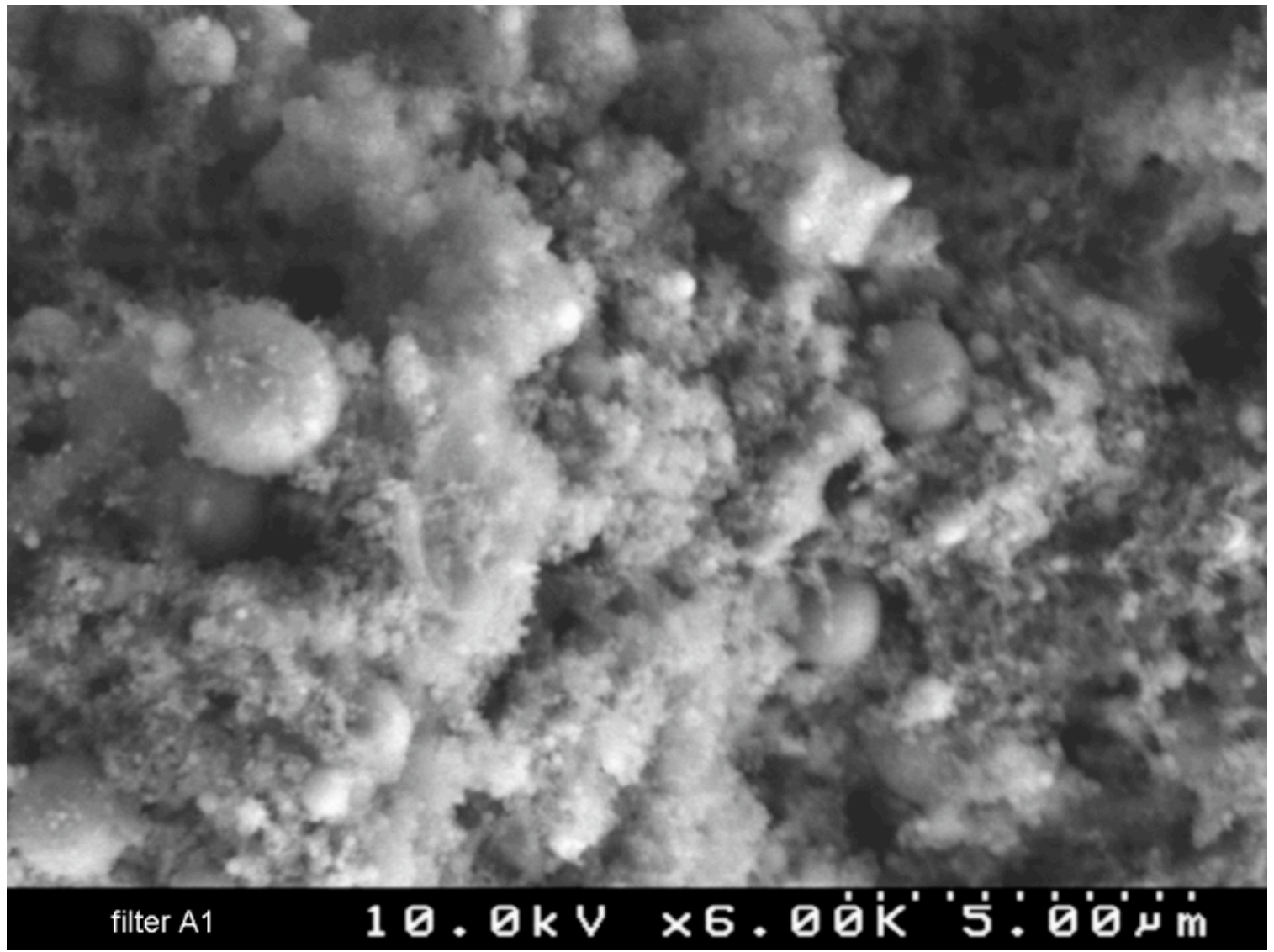

Label A: filter A1, 1.0u dia spherical particle, 15KV

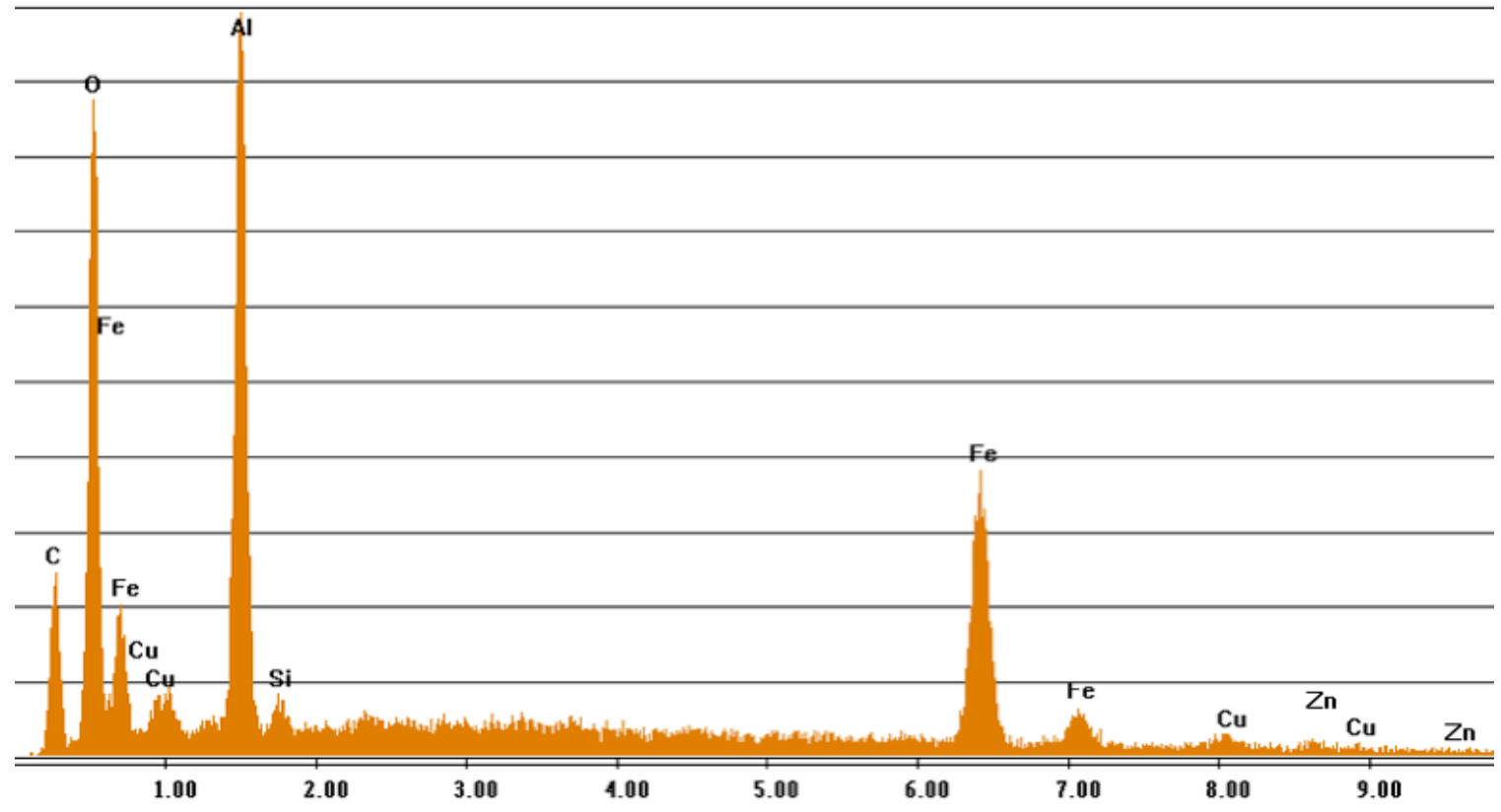


Figures 8A\&B; METS 04A Tank B; Collected next 5 minutes post-detonation at $\sim 20$ microns.

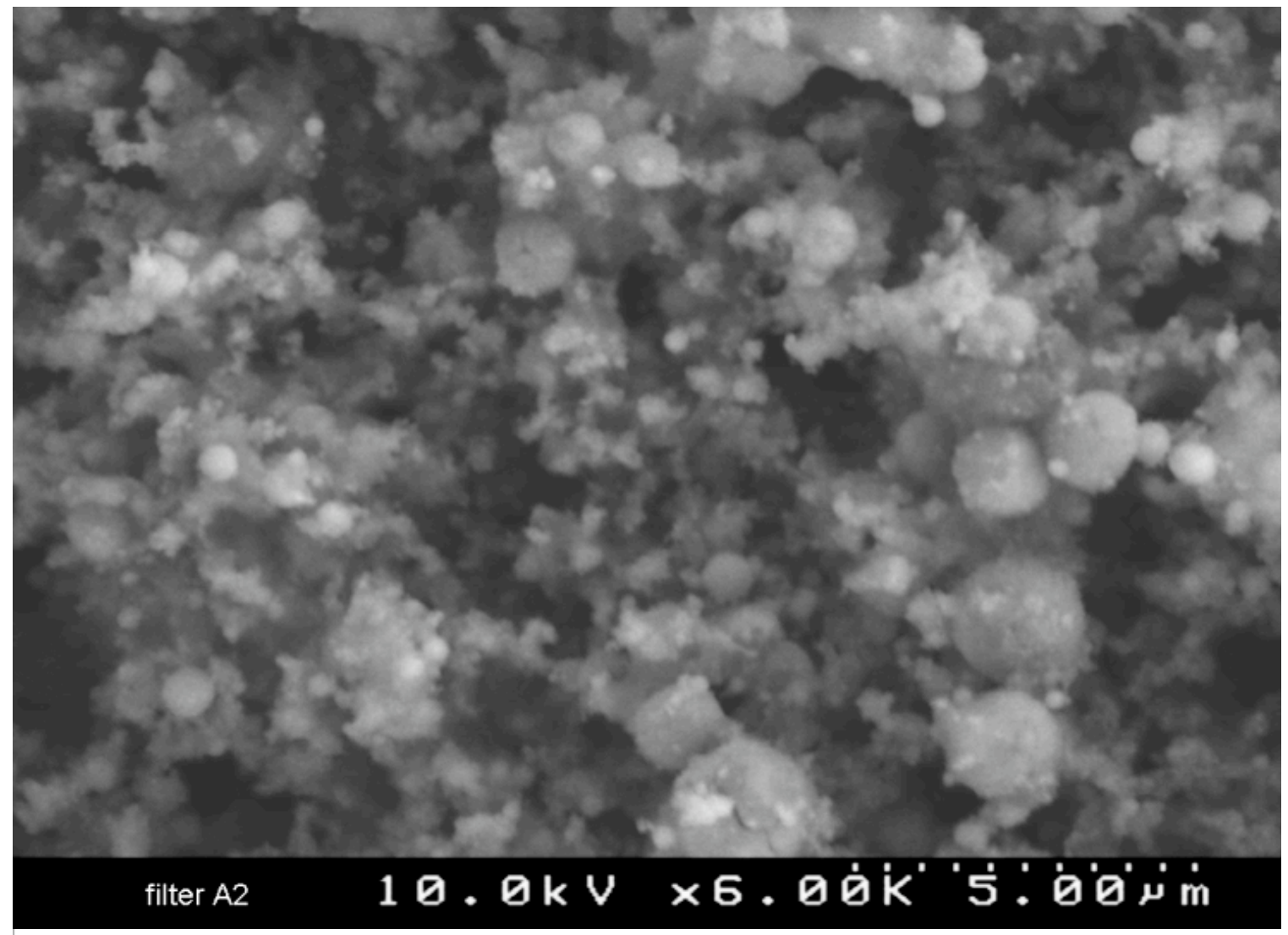

Label A: filter B1, 2.0u dia spherical particle, $15 \mathrm{KV}$

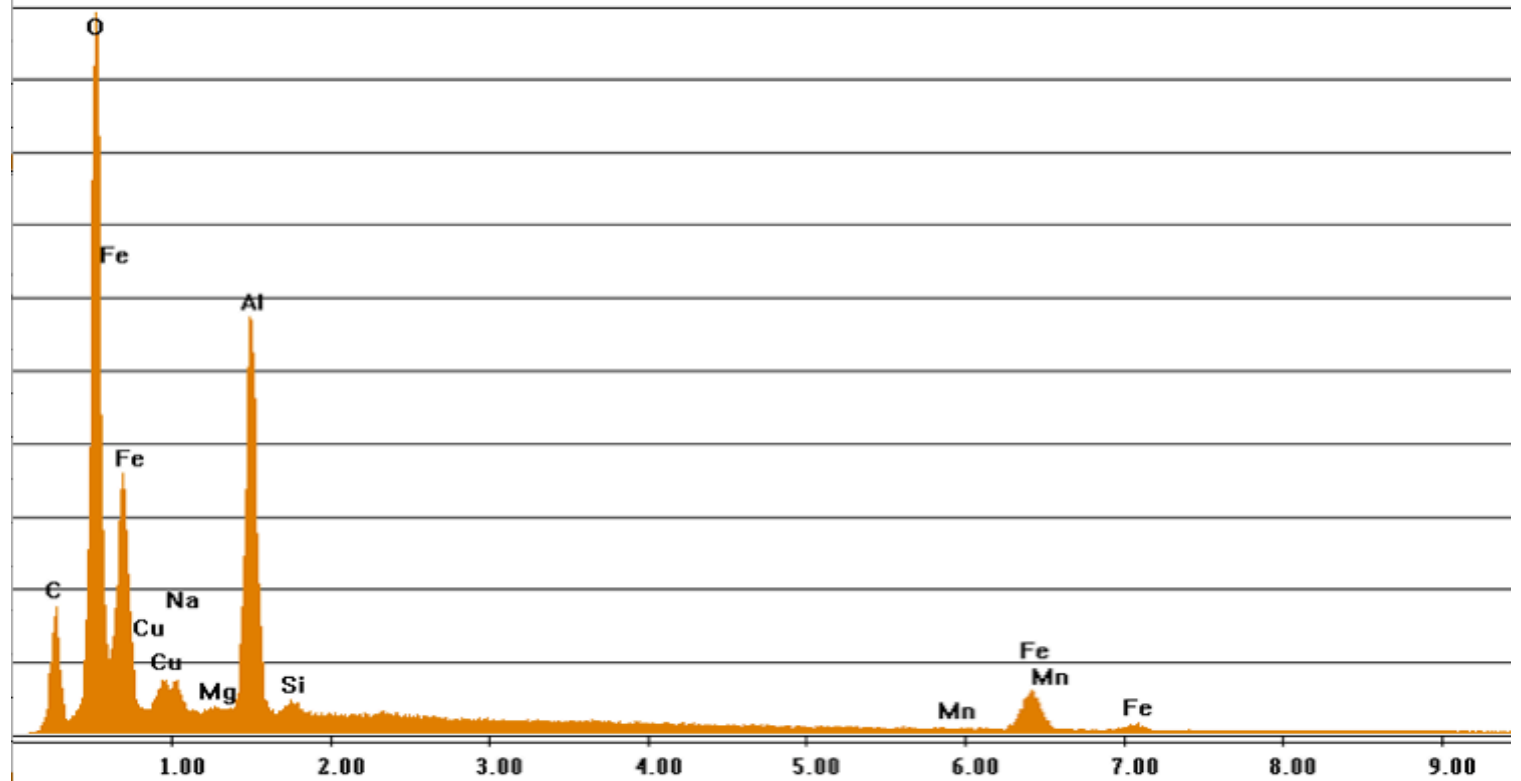


Figure 9A\&B; METS 05A Tank A; Collected 15 seconds post detonation, 20 microns

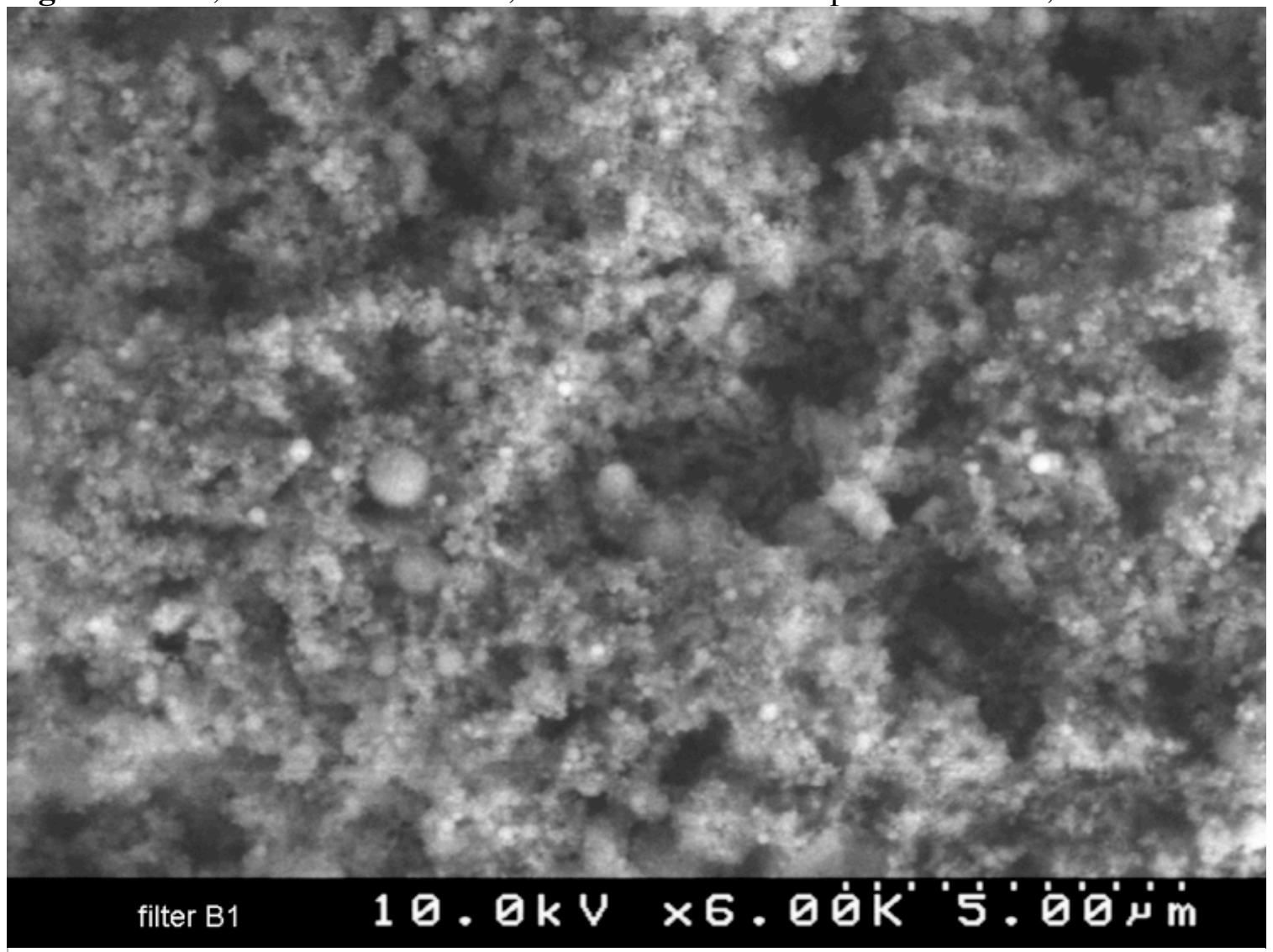

Label A: filter B1, filter surface deposit, 6KV

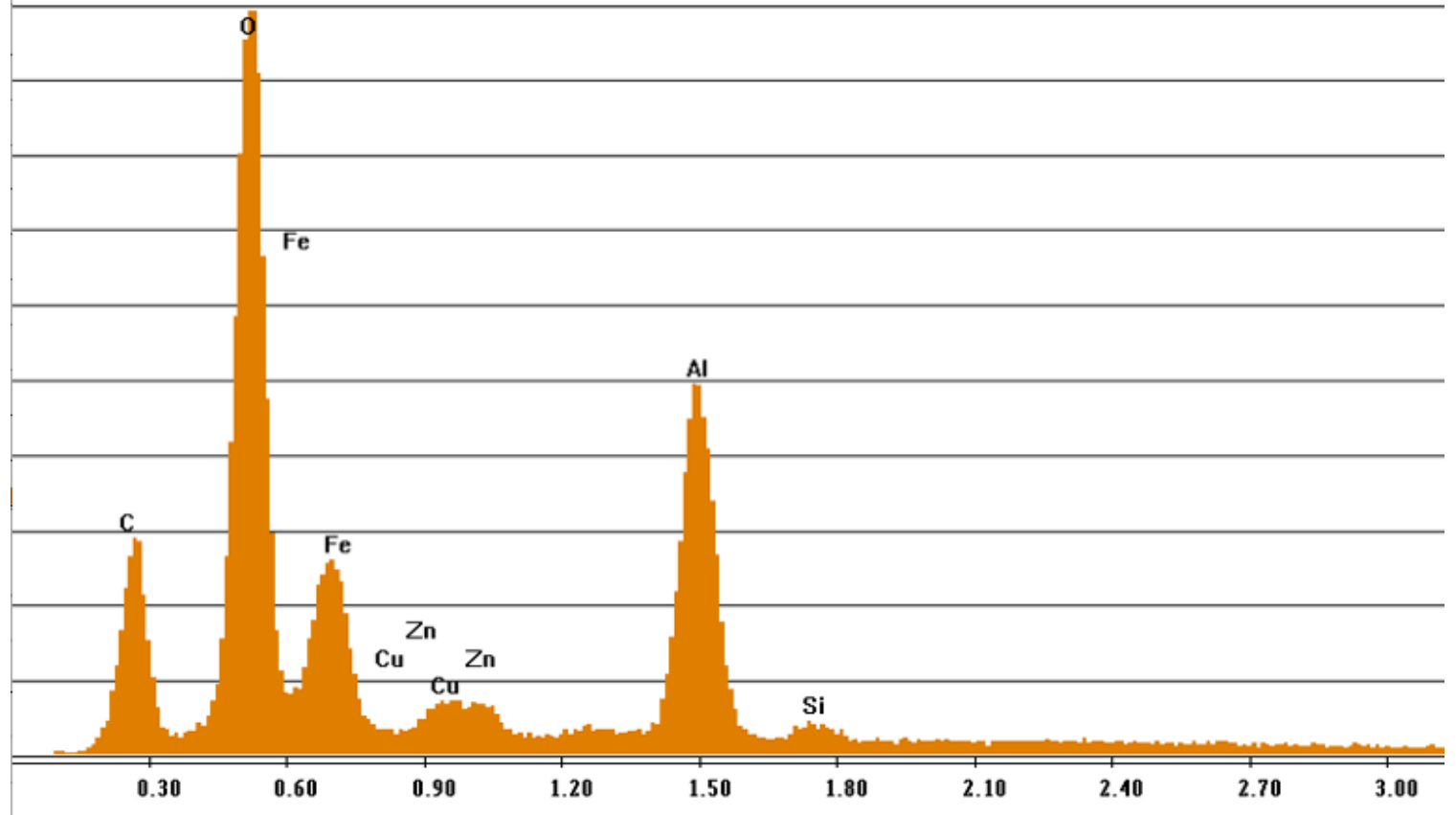


Figure 10A\&B; METS 05A Tank A; Collected 15 seconds post detonation at $\sim 4$ microns

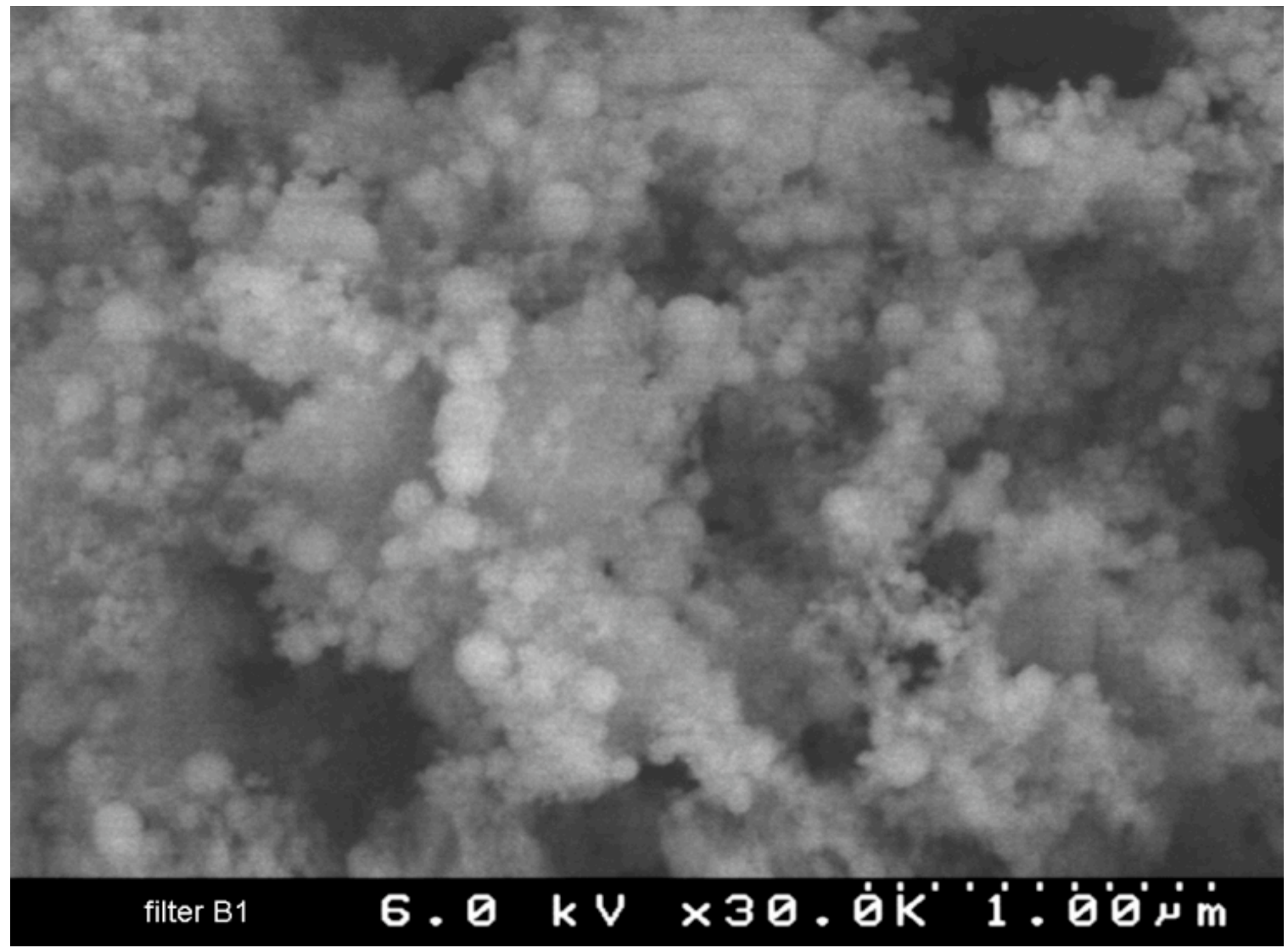

Label A: filter B1, <100nm predominantly spherical particles, 6KV

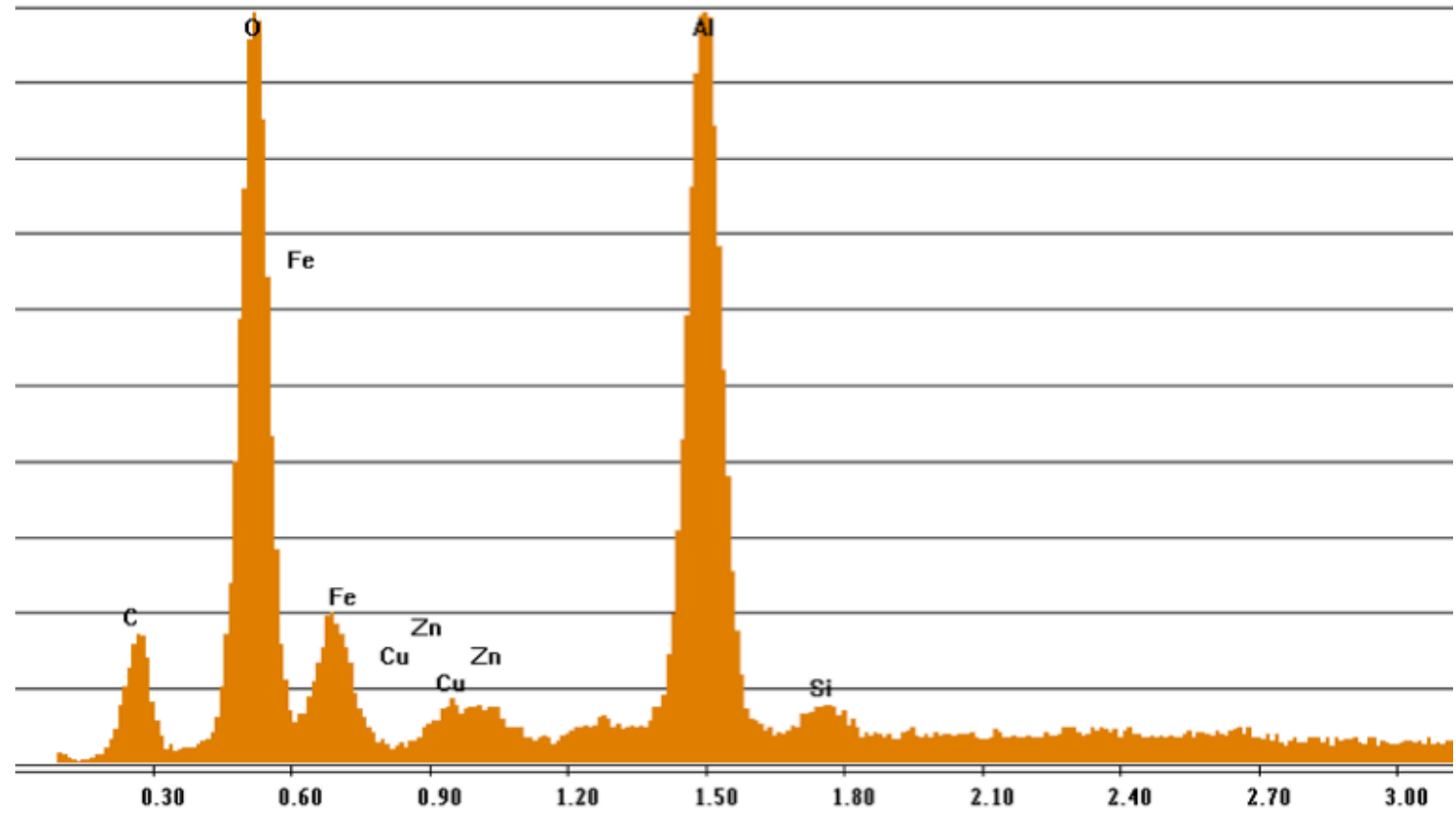




\section{Cascade Impactors}

After a minimum of 24 hours post-detonation the firing tank door is opened for the first time after the particulate has been given the same opportunity to settle as prescribed within the CFF chamber procedures. Placed inside the firing tank at this time are duplicate cascade impactors, offering size fractions in the respirable range $(<10$ microns) for the comparison of total beryllium and beryllium metal. The cascade impactor samples were taken simultaneously in a homogenously mixed internal tank environment. Mixing utilized a hollow metal tube system, with 10 holes along its bottom, to remotely distribute 60 psi hydraulically driven air across an approximate 3 foot by 1 foot area of the closed firing tank floor's surface for a specified period of time. The mixing system and the cascade impactors ran for 5 minutes during METS 04A and both ran for 20 minutes during METS 05A and 06A. The cascade impactors and the mixing systems were then turned off. Results for these cascade impactors are presented in Table 2.

Table 2; Cascade impactor results for each stage as both total beryllium and beryllium metal from METS 04A (Batch \#s 20080171 \& 2008172, Field Sample \#s 2111523, 3032739 - 746 \& 2111522, $303731-738$ ), METS 05A (Batch \#'s 20080340 \& 20080435, Field Sample \#s $2111619-627 \& 2111658-663^{*}$ ), and METS 06A (Batch \#'s 20080607 \& 20080608, Field Sample \#s 2111731 - 739 \& 2111740 - 748).

\begin{tabular}{|c|c|c|c|}
\hline $\begin{array}{c}\text { Cascade Impactor } \\
\begin{array}{c}\text { Size Fraction } \\
\text { (microns) }\end{array}\end{array}$ & $\begin{array}{c}\text { METS 04A } \\
\text { Total Be / Be Metal } \\
\mu \mathrm{g} / \mathrm{m}^{3}\end{array}$ & $\begin{array}{c}\text { METS 05A } \\
\text { Total Be/Be Metal } \\
\mu \mathrm{g} / \mathrm{m}^{3}\end{array}$ & $\begin{array}{c}\text { METS 06A } \\
\text { Total Be } / \text { Be Metal } \\
\mu \mathrm{g} / \mathrm{m}^{3 * * * *}\end{array}$ \\
\hline 10 to 9 & $28 / 23$ & $0.14 / 0.15$ & $0.088 / 0.041$ \\
\hline 9 to 5.8 & $29 / 32$ & $0.26 / 0.30$ & $0.12 / 0.076$ \\
\hline 5.8 to 4.7 & $23 / 20$ & $0.29 / 0.32$ & $0.12 / 0.073$ \\
\hline 4.7 to 3.3 & $35 / 40$ & 0.57 & $0.27 / 0.2$ \\
\hline 3.3 to 2.1 & $44 / 45$ & 0.68 & $0.29 / 0.36$ \\
\hline 2.1 to 1.1 & $27 / 28$ & $0.42 / 0.32$ & $0.19 / 0.19$ \\
\hline 1.1 to 0.65 & $2.6 / 2.8$ & $0.065 / 0.057$ & $<0.035 /<0.035$ \\
\hline 0.65 to 0.43 & $0.77 / 0.49$ & $0.11 / 0.14$ & $<0.035 /<0.035$ \\
\hline \hline $\begin{array}{c}\text { Respirable Particulate } \\
\text { Mass } \\
(<10)^{* * *}\end{array}$ & $190 / 191$ & $2.5 / * * * *$ & $1.1 / 0.94$ \\
\hline
\end{tabular}

$* \mu \mathrm{g} / \mathrm{m}^{3} ;$ micrograms per cubic meter of air.

** Cascade impactor stages separated with only six retained intact, beryllium metal results are therefore estimated for similarity in size fraction to the total beryllium result. *** Additive respirable particulate mass (ACGIH TLV 2005) derived from the total of the individual stages.

**** Additive respirable particulate mass not possible due to incomplete beryllium metal results. 


\section{Chamber Environment Personal Air and Surrogates}

Actual personal sampling monitoring results and chamber environment comparable monitoring devices that are obtained with equipment and at locations indicative of personal sampling levels are presented in the tables below.

Personal Sampling

Table 3; Personal air sampling for Total Beryllium during METS 04 open firing tank procedures on 8/17/05 covered under Phase 1 of HAC_812_METS04-06_Be.doc in PAPR full face respirators with P100 cartridges, tyvek and gloves, booties from Batch \# 20080183 are as follows:

\begin{tabular}{|c|c|c|}
\hline Employee & Sample Result & $\begin{array}{c}\text { Calculated 8-Hr Time } \\
\text { Weighted Average (TWA) }\end{array}$ \\
\hline $\begin{array}{c}\text { Employee A } \\
\text { Emp \# xxxxxx } \\
\text { Sample and Equipment Retrieval } \\
\text { FS\#2111532 }\end{array}$ & $\begin{array}{c}0.3 \mu \mathrm{g} / \mathrm{m}^{3} \\
\text { (35 minutes) }\end{array}$ & $0.022 \mu \mathrm{g} / \mathrm{m}^{3}$ \\
\hline $\begin{array}{c}\text { Employee B } \\
\text { Emp \# xxxxxx } \\
\text { Sample and Equipment Retrieval } \\
\text { FS\#2111533 }\end{array}$ & $\begin{array}{c}0.051 \mu \mathrm{g} / \mathrm{m}^{3} \\
(192 \text { minutes })\end{array}$ & $0.020 \mu \mathrm{g} / \mathrm{m}^{3}$ \\
\hline $\begin{array}{c}\text { Employee C } \\
\text { Emp \# xxxxxx } \\
\text { ES\&H and Sampling Assistance } \\
\text { FS\#2111534 }\end{array}$ & $\begin{array}{l}0.12 \mu \mathrm{g} / \mathrm{m}^{3} \\
(67 \text { minutes })\end{array}$ & $0.017 \mu \mathrm{g} / \mathrm{m}^{3}$ \\
\hline Beryllium Action Level & --- & $0.2 \mu \mathrm{g} / \mathrm{m}^{3}$ \\
\hline
\end{tabular}

Table 4; Personal air sampling for Total Beryllium during METS 04 open firing tank procedures on 8/18/05, covered under Phases 1 and 2 of HAC_812_METS04-06_Be.doc in PAPR full face respirators with P100 cartridges, tyvek and gloves, booties from Batch \#s 20080185 and 20080177 are as follows:

\begin{tabular}{|c|c|c|c|}
\hline Employee & $\begin{array}{c}\text { Sample Result } \\
\text { a.m. }\end{array}$ & $\begin{array}{c}\text { Sample Result } \\
\text { p.m. }\end{array}$ & $\begin{array}{c}\text { Calculated 8-Hr } \\
\text { TWA }\end{array}$ \\
\hline $\begin{array}{c}\text { Employee B } \\
\text { Emp \# xxxxxx } \\
\text { Sampling \& Cleaning } \\
\text { FS\#2111536, 2111502 }\end{array}$ & $\begin{array}{c}0.58 \mu \mathrm{g} / \mathrm{m}^{3} \\
(67 \text { minutes })\end{array}$ & $\begin{array}{c}0.21 \mu \mathrm{g} / \mathrm{m}^{3} \\
(45 \mathrm{minutes})\end{array}$ & $0.10 \mu \mathrm{g} / \mathrm{m}^{3}$ \\
\hline Employee D & $\begin{array}{c}0.61 \mu \mathrm{g} / \mathrm{m}^{3} \\
\text { Emp \# xxxxxx }\end{array}$ & $\begin{array}{c}0.17 \mu \mathrm{g} / \mathrm{m}^{3} \\
(45 \mathrm{minutes})\end{array}$ & $0.099 \mu \mathrm{g} / \mathrm{m}^{3}$ \\
Sampling \& Cleaning & $(65$ minutes $)$ & & \\
FS\#2111537, 2111503 & N/A & $0.02 \mu \mathrm{g} / \mathrm{m}^{3}$ \\
\hline Employee C & $0.32 \mu \mathrm{g} / \mathrm{m}^{3}$ & & \\
Emp \# xxxxxx & $(30$ minutes $)$ & & $0.2 \mu \mathrm{g} / \mathrm{m}^{3}$ \\
\hline ES\&H Assistance & --- & & \\
FS\#2111538 & & & \\
\hline Beryllium Action Level & & &
\end{tabular}


Table 5; Personal air sampling for Total Beryllium during METS 05 open firing tank procedures on 8/25/05, covered under Phases 1 and 2 of HAC_812_METS04-06_Be.doc in PAPR full face respirators with P100 cartridges, tyvek and gloves, booties from Batch \#s 20080323 and 20080316 are as follows:

\begin{tabular}{|c|c|c|c|}
\hline Employee & $\begin{array}{l}\text { Sample Result } \\
\text { a.m. }\end{array}$ & $\begin{array}{l}\text { Sample Result } \\
\text { p.m. }\end{array}$ & $\begin{array}{c}\text { Calculated } 8-\mathrm{Hr} \\
\text { TWA }\end{array}$ \\
\hline $\begin{array}{c}\text { Employee B } \\
\text { Emp \# xxxxxx } \\
\text { Sampling \& Cleaning } \\
\text { FS\#2111552, } 2111549\end{array}$ & $\begin{array}{c}<0.086 \mu \mathrm{g} / \mathrm{m}^{3} \\
(80 \text { minutes })\end{array}$ & $\begin{array}{l}<0.22 \mu \mathrm{g} / \mathrm{m}^{3} \\
(30 \text { minutes })\end{array}$ & $<0.014 \mu \mathrm{g} / \mathrm{m}^{3}$ \\
\hline $\begin{array}{c}\text { Employee D } \\
\text { Emp \# xxxxxx } \\
\text { Sampling \& Cleaning } \\
\text { FS\#2111553, 2111550 }\end{array}$ & $\begin{array}{l}<0.11 \mu \mathrm{g} / \mathrm{m}^{3} \\
(60 \text { minutes })\end{array}$ & $\begin{array}{c}0.11 \mu \mathrm{g} / \mathrm{m}^{3} \\
\text { (121 minutes) }\end{array}$ & $<0.041 \mu \mathrm{g} / \mathrm{m}^{3}$ \\
\hline $\begin{array}{c}\text { Employee E } \\
\text { Emp \# xxxxxx } \\
\text { Sampling \& Cleaning } \\
\text { FS\#2111555, } 2111547\end{array}$ & $\begin{array}{c}0.14 \mu \mathrm{g} / \mathrm{m}^{3} \\
(53 \text { minutes })\end{array}$ & $\begin{array}{c}<0.078 \mu \mathrm{g} / \mathrm{m}^{3} \\
(86 \text { minutes })\end{array}$ & $<0.029 \mu \mathrm{g} / \mathrm{m}^{3}$ \\
\hline $\begin{array}{c}\text { Employee F } \\
\text { Emp \# xxxxxx } \\
\text { Cleaning } \\
\text { FS\#2111548 } \\
\end{array}$ & -- & $\begin{array}{c}<0.2 \mu \mathrm{g} / \mathrm{m}^{3} \\
(35 \text { minutes })\end{array}$ & $<0.014 \mu \mathrm{g} / \mathrm{m}^{3}$ \\
\hline $\begin{array}{c}\text { Employee G } \\
\text { Emp \# xxxxxx } \\
\text { Cleaning } \\
\text { FS\#2111554 }\end{array}$ & $\begin{array}{l}<0.11 \mu \mathrm{g} / \mathrm{m}^{3} \\
(58 \text { minutes })\end{array}$ & --- & $<0.014 \mu \mathrm{g} / \mathrm{m}^{3}$ \\
\hline Beryllium Action Level & --- & & $0.2 \mu \mathrm{g} / \mathrm{m}^{3}$ \\
\hline
\end{tabular}

Table 6; Personal air sampling for Total Beryllium during METS 05 open firing tank procedures on 8/29/05 covered under Phase 2 of HAC 812 METS04-06_Be.doc in PAPR full face respirators with P100 cartridges, tyvek and gloves, booties from Batch \# 20080385 are as follows:

\begin{tabular}{|c|c|c|}
\hline Employee & Sample Result & $\begin{array}{c}\text { Calculated 8-Hr Time } \\
\text { Weighted Average (TWA) }\end{array}$ \\
\hline Employee B & $\begin{array}{c}<0.17 \mu \mathrm{g} / \mathrm{m}^{3} \\
(41 \mathrm{minutes})\end{array}$ & $<0.014 \mu \mathrm{g} / \mathrm{m}^{3}$ \\
$\begin{array}{c}\text { Emp \# xxxxxx } \\
\text { Cleaning } \\
\text { FS\#2111652 }\end{array}$ & $\begin{array}{c}<0.18 \mu \mathrm{g} / \mathrm{m}^{3} \\
(38 \mathrm{minutes})\end{array}$ & $<0.015 \mu \mathrm{g} / \mathrm{m}^{3}$ \\
\hline Employee D & & \\
Emp \# xxxxxx & --- & $0.2 \mu \mathrm{g} / \mathrm{m}^{3}$ \\
Cleaning & & \\
\hline FS\#2111653 & &
\end{tabular}


Table 7; Personal air sampling for Total Beryllium during METS 05 open firing tank procedures on 8/30/05 covered under Phase 3 of HAC_812_METS04-06_Be.doc in PAPR full face respirators with P100 cartridges, tyvek and gloves, booties from Batch \# 20080400 are as follows:

\begin{tabular}{|c|c|c|}
\hline Employee & Sample Result & $\begin{array}{c}\text { Calculated 8-Hr Time } \\
\text { Weighted Average (TWA) }\end{array}$ \\
\hline Employee B & $<0.24 \mu \mathrm{g} / \mathrm{m}^{3}$ \\
Emp \# xxxxxx & $\begin{array}{c}<0.015 \mu \mathrm{g} / \mathrm{m}^{3} \\
(30 \text { minutes) }\end{array}$ & \\
METS 06A Shot Set_Up & & $<0.014 \mu \mathrm{g} / \mathrm{m}^{3}$ \\
FS\#2111655 & $<0.16 \mu \mathrm{g} / \mathrm{m}^{3}$ & \\
Employee D & $(42 \mathrm{minutes})$ & \\
Emp \# xxxxxx & & $0.2 \mu \mathrm{g} / \mathrm{m}^{3}$ \\
\hline FETS 06A Shot Set_Up & --- & \\
\hline Beryllium Action Level & & \\
\hline
\end{tabular}

Table 8; Personal air sampling for Total Beryllium during METS 06A open firing tank procedures on 9/06/05, covered under Phases 1 and 2 of HAC_812_METS04-06_Be.doc in PAPR full face respirators with P100 cartridges, tyvek and gloves, booties from Batch \#s 20080564 and 20080580 are as follows:

\begin{tabular}{|c|c|c|c|}
\hline Employee & $\begin{array}{c}\text { Sample Result } \\
\text { a.m. }\end{array}$ & $\begin{array}{c}\text { Sample Result } \\
\text { p.m. }\end{array}$ & $\begin{array}{c}\text { Calculated 8-Hr } \\
\text { TWA }\end{array}$ \\
\hline $\begin{array}{c}\text { Employee B } \\
\text { Emp \# xxxxxx } \\
\text { Sampling \& Cleaning } \\
\text { FS\#2111678, 2111682 }\end{array}$ & $\begin{array}{c}<0.037 \mu \mathrm{g} / \mathrm{m}^{3} \\
(179 \text { minutes })\end{array}$ & $\begin{array}{c}<0.18 \mu \mathrm{g} / \mathrm{m}^{3} \\
(56 \text { minutes })\end{array}$ & $<0.015 \mu \mathrm{g} / \mathrm{m}^{3}$ \\
\hline Employee D & & & \\
Emp \# xxxxxx & $<0.064 \mu \mathrm{g} / \mathrm{m}^{3}$ & $<0.15 \mu \mathrm{g} / \mathrm{m}^{3}$ & $<0.014 \mu \mathrm{g} / \mathrm{m}^{3}$ \\
Sampling \& Cleaning & $(104 \mathrm{minutes})$ & $(44 \mathrm{minutes})$ & \\
FS\#2111677, 2111681 & & --- & $<0.014 \mu \mathrm{g} / \mathrm{m}^{3}$ \\
\hline Employee C & $<0.09 \mu \mathrm{g} / \mathrm{m}^{3}$ & & \\
Emp \# xxxxxx & $(72 \mathrm{minutes})$ & & $0.2 \mu \mathrm{g} / \mathrm{m}^{3}$ \\
\hline Sampling \& Cleaning & & & \\
FS\#2111679 & --- & & \\
\hline Beryllium Action Level & & & \\
\hline
\end{tabular}


Table 9; Personal air sampling for Total Beryllium during METS 06A open firing tank procedures on 9/14/05 covered under Phase 2 of HAC_812_METS04-06_Be.doc in PAPR full face respirators with P100 cartridges, tyvek and gloves, booties from Batch \# 20080765 are as follows:

\begin{tabular}{|c|c|c|}
\hline Employee & Sample Result & $\begin{array}{c}\text { Calculated 8-Hr Time } \\
\text { Weighted Average (TWA) }\end{array}$ \\
\hline Employee B & $\begin{array}{c}<0.11 \mu \mathrm{g} / \mathrm{m}^{3} \\
(53 \text { minutes) }\end{array}$ & $<0.012 \mu \mathrm{g} / \mathrm{m}^{3}$ \\
$\begin{array}{c}\text { Emp \# xxxxxx } \\
\text { Cleaning } \\
\text { FS\#2111783 }\end{array}$ & $\begin{array}{c}<0.15 \mu \mathrm{g} / \mathrm{m}^{3} \\
(44 \mathrm{minutes})\end{array}$ & $<0.014 \mu \mathrm{g} / \mathrm{m}^{3}$ \\
\hline Employee D & & \\
Emp \# xxxxxx & --- & $0.2 \mu \mathrm{g} / \mathrm{m}^{3}$ \\
Cleaning & & \\
\hline FS\#2111784 & &
\end{tabular}

\section{Tank Environment Sampling}

Table 10; Internal firing tank air sampling, to replicate personal sampling parameters, for Total Beryllium during METS 04A - 06A. As these samples are taken with personal air sampling pumps, calibrated at typical personal monitoring levels and fitted with standard total dust cassettes, they can be directly compared to the Beryllium Action Level. Presented below are results from Batch \#s 20080174 (FS\# 2111527), 20080337 (FS\# 2111617), and 20080177 and are as follows:

\begin{tabular}{|c|c|c|c|}
\hline METS Test & METS 04A & METS 05A. & METS 06A \\
\hline $\begin{array}{l}\text { Homogenously Mixed } \\
\text { Internal Environment at } \\
\text { Breathing Zone Level } \\
\text { (Standard Total Dust) }\end{array}$ & $\begin{array}{c}41 \mu \mathrm{g} / \mathrm{m}^{3} \\
\text { (106 minutes) }\end{array}$ & $\begin{array}{c}1.9 \mu \mathrm{g} / \mathrm{m}^{3} \\
\text { (76 minutes) }\end{array}$ & $\begin{array}{c}1.0 \mu \mathrm{g} / \mathrm{m}^{3} \\
\text { (89 minutes) }\end{array}$ \\
\hline Beryllium Action Level & $0.2 \mu \mathrm{g} / \mathrm{m}^{3}$ & $0.2 \mu \mathrm{g} / \mathrm{m}^{3}$ & $0.2 \mu \mathrm{g} / \mathrm{m}^{3}$ \\
\hline
\end{tabular}


Table 11; Internal firing tank size-selective air sampling, to replicate personal sampling parameters, for Beryllium Metal during METS 04A - 06A and were taken at the same time as like experimental results presented in Table 10. The results below were analyzed at DataChem Analytical Laboratory and belong to a unique experimental batch for NIOSH/LANL Project ID DART 06-7225 with the results are as follows:

\begin{tabular}{|c|c|c|}
\hline Size-Selective Sampling Device & METS 05A & METS 06A \\
\hline $\begin{array}{c}\text { GSA-GSP (Inhalable at Breathing Zone) } \\
\text { Internal Tank Homogeneously Mixed } \\
\text { FS\#10435 }\end{array}$ & (Pump not available) & $\begin{array}{c}0.83 \mu \mathrm{g} / \mathrm{m}^{3} \\
\text { (72 Minutes) }\end{array}$ \\
\hline $\begin{array}{c}\text { GSA-GSP (Inhalable at Breathing Zone) } \\
\text { Internal Tank Homogeneously Mixed } \\
\text { FS\#10436 } \\
\end{array}$ & (Pump not available) & $\begin{array}{c}1.4 \mu \mathrm{g} / \mathrm{m}^{3} \\
\text { (71 Minutes) } \\
\end{array}$ \\
\hline $\begin{array}{c}\text { CIP-10 (Inhalable at Floor Level) } \\
\text { Internal Tank Homogeneously Mixed } \\
\text { FS\#10437, } 10438\end{array}$ & $\begin{array}{c}1.1 \mu \mathrm{g} / \mathrm{m}^{3} \\
\text { (82 Minutes) }\end{array}$ & $\begin{array}{c}0.37 \mu \mathrm{g} / \mathrm{m}^{3} \\
\text { (78 Minutes) }\end{array}$ \\
\hline $\begin{array}{c}\text { IOM (Inhalable at Breathing Zone) } \\
\text { Internal Tank Homogeneously Mixed } \\
\text { FS\#10439, } 10441 \\
\end{array}$ & $\begin{array}{c}1.5 \mu \mathrm{g} / \mathrm{m}^{3} \\
\text { (78 Minutes) }\end{array}$ & $\begin{array}{c}0.96 \mu \mathrm{g} / \mathrm{m}^{3} \\
\text { (99 Minutes) }\end{array}$ \\
\hline $\begin{array}{c}\text { IOM (Inhalable at Breathing Zone) } \\
\text { Internal Tank Homogeneously Mixed } \\
\text { FS\#10443 }\end{array}$ & $\begin{array}{c}\text { (Duplicate pump not } \\
\text { available) }\end{array}$ & $\begin{array}{c}0.72 \mu \mathrm{g} / \mathrm{m}^{3} \\
\text { (97 Minutes) }\end{array}$ \\
\hline $\begin{array}{c}\text { IOM (Respirable at Breathing Zone) } \\
\text { Internal Tank Homogeneously Mixed } \\
\text { FS\#10440, } 10442 \\
\end{array}$ & $\begin{array}{c}0.77 \mu \mathrm{g} / \mathrm{m}^{3} \\
\text { (78 Minutes) }\end{array}$ & $\begin{array}{c}0.39 \mu \mathrm{g} / \mathrm{m}^{3} \\
\text { (99 Minutes) }\end{array}$ \\
\hline $\begin{array}{c}\text { IOM (Respirable at Breathing Zone) } \\
\text { Internal Tank Homogeneously Mixed } \\
\text { FS\#10444 }\end{array}$ & $\begin{array}{c}\text { (Duplicate pump not } \\
\text { available) }\end{array}$ & $\begin{array}{c}0.31 \mu \mathrm{g} / \mathrm{m}^{3} \\
\text { (97 Minutes) }\end{array}$ \\
\hline
\end{tabular}

* As these sampling devices are size-selective, they cannot be compared to the existing Beryllium Action Limit or the Permissible Exposure Limit as these are based on standard total dust cassettes which are not size-selective by design.

\section{Surface Swipe Sampling}

Investigative surface swipe sampling was performed on the firing tank's surfaces to indicate representative surface levels for total beryllium. Initial results presented were all taken wet (Table 12, Figures $11-14$ ) prior to the cleaning of the tank's interior. These wet surface swipes were taken after a minimum of 24 hours after METS 05A and 06A detonation and after the settling of the tank's internal environment subsequent to the removal of sampling devices relating to the monitoring protocol during the chamber's homogenous mixing. The means of the pre-cleaning floor and wall surface wet swiping protocol, with each swipe taken over a 100 square centimeter area, are presented for comparison in Table 12 and the individual surface swipe sample results in relation to their pre-cleaning mean are presented in Figures 11 - 14. Results presented in the figures are obtained from analytical batch numbers 20080336 and 20080589 with the field samples numbers respectively $2111587-2111607$ and $2111699-2111713$. A 
comparison of wet swiping to dry swiping protocol are presented in Tables $13-15$ and were taken post shot and prior to cleaning, as well as after tank cleaning procedures.

Table 12; Means of the surface wet swipes taken on the firing tank floor and wall, post detonation and pre-cleaning, for total beryllium (metal and oxide) for METS 05A and 06A experiments. Compare to Release Criterion of $0.002 \mu \mathrm{g} / \mathrm{cm}^{2}$ as Total Beryllium.

\begin{tabular}{|c|c|c|}
\hline $\begin{array}{c}\text { Sample Sequence } \\
\text { Batch \# } \\
\text { Sample \# }\end{array}$ & $\begin{array}{c}\text { Mean of Floor Surfaces } \\
\text { Total Beryllium } \\
\mu \mathrm{g} / \mathrm{cm}^{2} *\end{array}$ & $\begin{array}{c}\text { Mean of Wall Surfaces } \\
\text { Total Beryllium } \\
\mu \mathrm{g} / \mathrm{cm}^{2} *\end{array}$ \\
\hline $\begin{array}{c}\text { 8/25/05 } \\
\text { METS 05A Pre-Cleaning } \\
\text { Surfaces } \\
\text { Batch\# 20080336 }\end{array}$ & 1.76 & \\
FS\#2111587-607 & & \\
\hline $\begin{array}{c}\text { 9/01/05 } \\
\text { METS 06A Pre-Cleaning } \\
\text { Surfaces }\end{array}$ & & \\
Batch\# 20080589 & 0.047 & 0.02 \\
FS\#21116999-713 & & \\
\hline
\end{tabular}

Table 13; Surface swipe results taken post-detonation, and pre-cleaning from METS 04A, to provide a side-by-side comparison of wet versus dry swipe sampling protocol. From Batch \# 20080078 on 8/18/05 are as follows:

\begin{tabular}{|c|c|c|}
\hline $\begin{array}{c}\text { Experimental Location } \\
\text { Sample \# }\end{array}$ & $\begin{array}{c}\text { Wet } \\
\mu \mathrm{g} / \mathrm{cm}^{2}\end{array}$ & $\begin{array}{c}\text { Dry } \\
\mu \mathrm{g} / \mathrm{cm}^{2}\end{array}$ \\
\hline $\begin{array}{c}\text { Pre-Cleaning Firing Tank Door } \\
\text { FS\#2111451 -52 }\end{array}$ & 0.24 & 0.01 \\
\hline $\begin{array}{c}\text { Pre-Cleaning Firing Tank Door } \\
\text { FS\#2111453-54 }\end{array}$ & 0.21 & 0.013 \\
\hline $\begin{array}{c}\text { Pre-Cleaning Firing Tank Floor } \\
\text { FS\#2111455-56 }\end{array}$ & 1.6 & 0.028 \\
\hline $\begin{array}{c}\text { Pre-Cleaning Firing Tank Floor } \\
\text { FS\#2111457-58 }\end{array}$ & 0.74 & 0.041 \\
\hline $\begin{array}{c}\text { Pre-Cleaning Firing Tank Wall } \\
\text { FS\#2111459-60 }\end{array}$ & 0.22 & 0.018 \\
\hline $\begin{array}{c}\text { Pre-Cleaning Firing Tank Wall } \\
\text { FS\#2111461-62 }\end{array}$ & 0.77 & 0.027 \\
\hline $\begin{array}{c}\text { Pre-Cleaning Firing Tank Ceiling } \\
\text { FS\#2111463, 2111465 }\end{array}$ & 0.033 & 0.0026 \\
\hline $\begin{array}{c}\text { Pre-Cleaning Firing Tank Ceiling } \\
\text { FS\#2111464, 2111466 }\end{array}$ & 0.03 & 0.0022 \\
\hline
\end{tabular}


Figure 11; Surface wet swipes (Table 12) of the firing tank floor prior to cleaning of the surfaces.

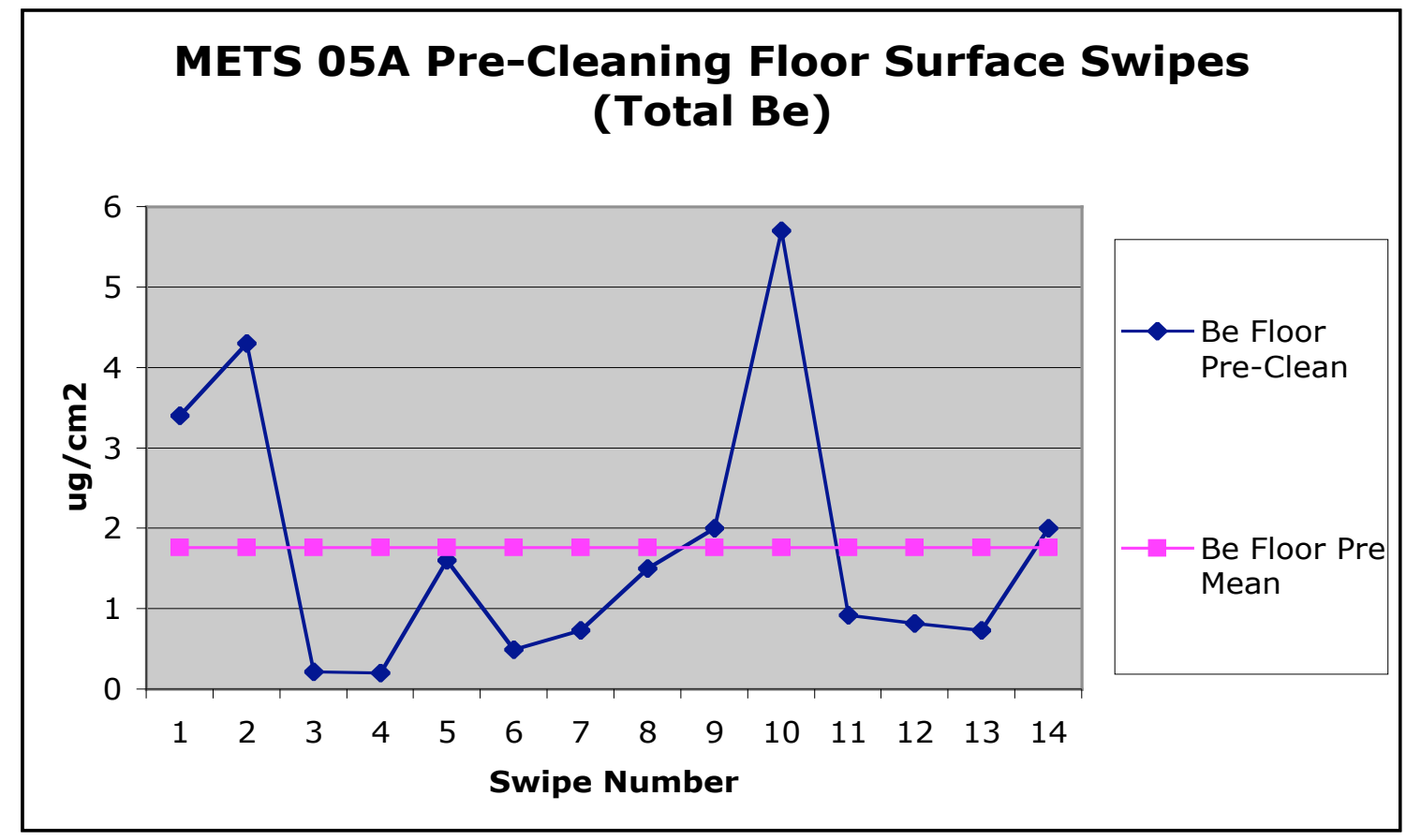

Figure 12; Surface wet swipes (Table 12) of the firing tank wall prior to cleaning of the surfaces.

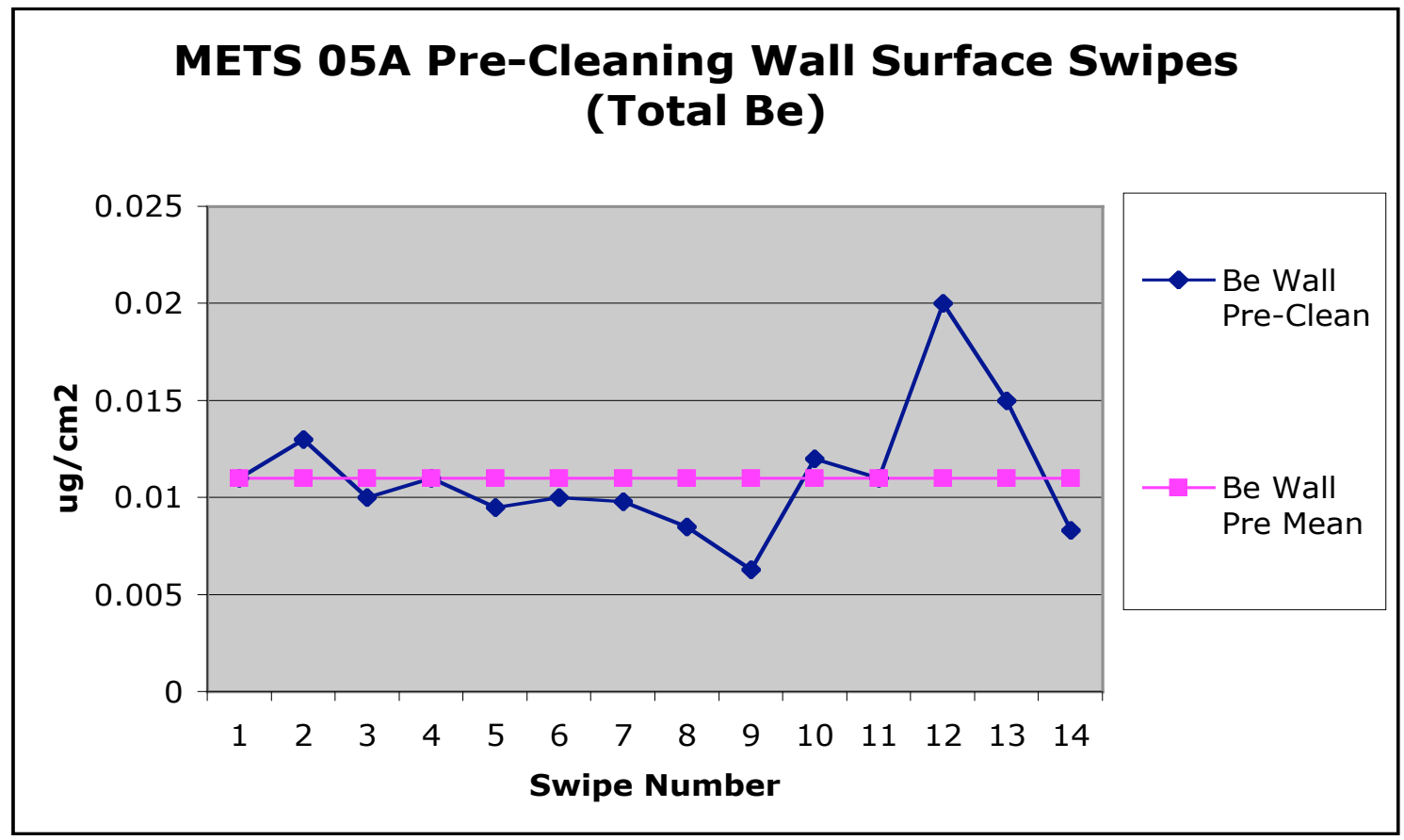


Figure 13; Surface wet swipes (Table 12) of the firing tank floor prior to cleaning of the surfaces.

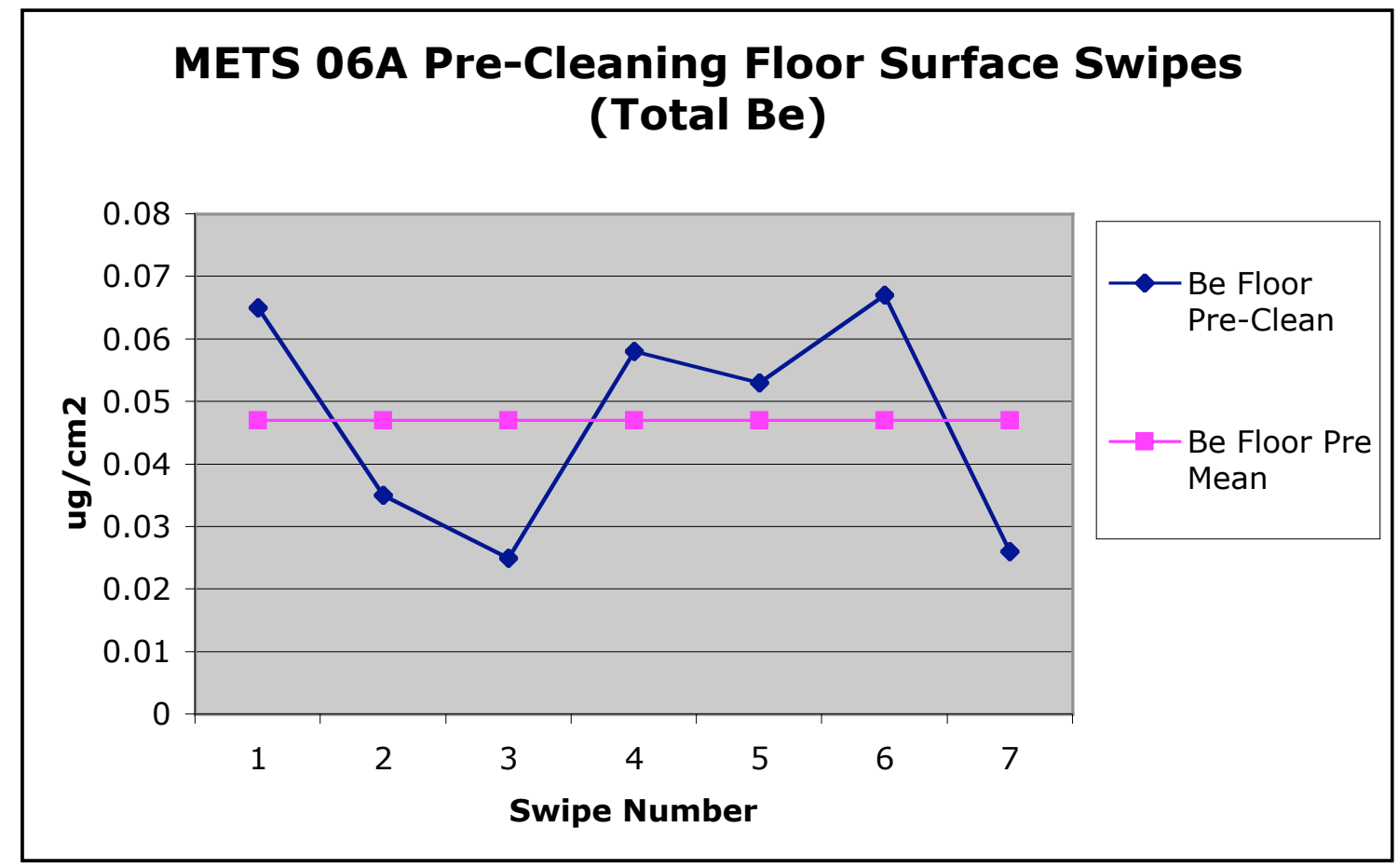

Figure 14; Surface wet swipes (Table 12) of the firing tank walls prior to cleaning of the surfaces.

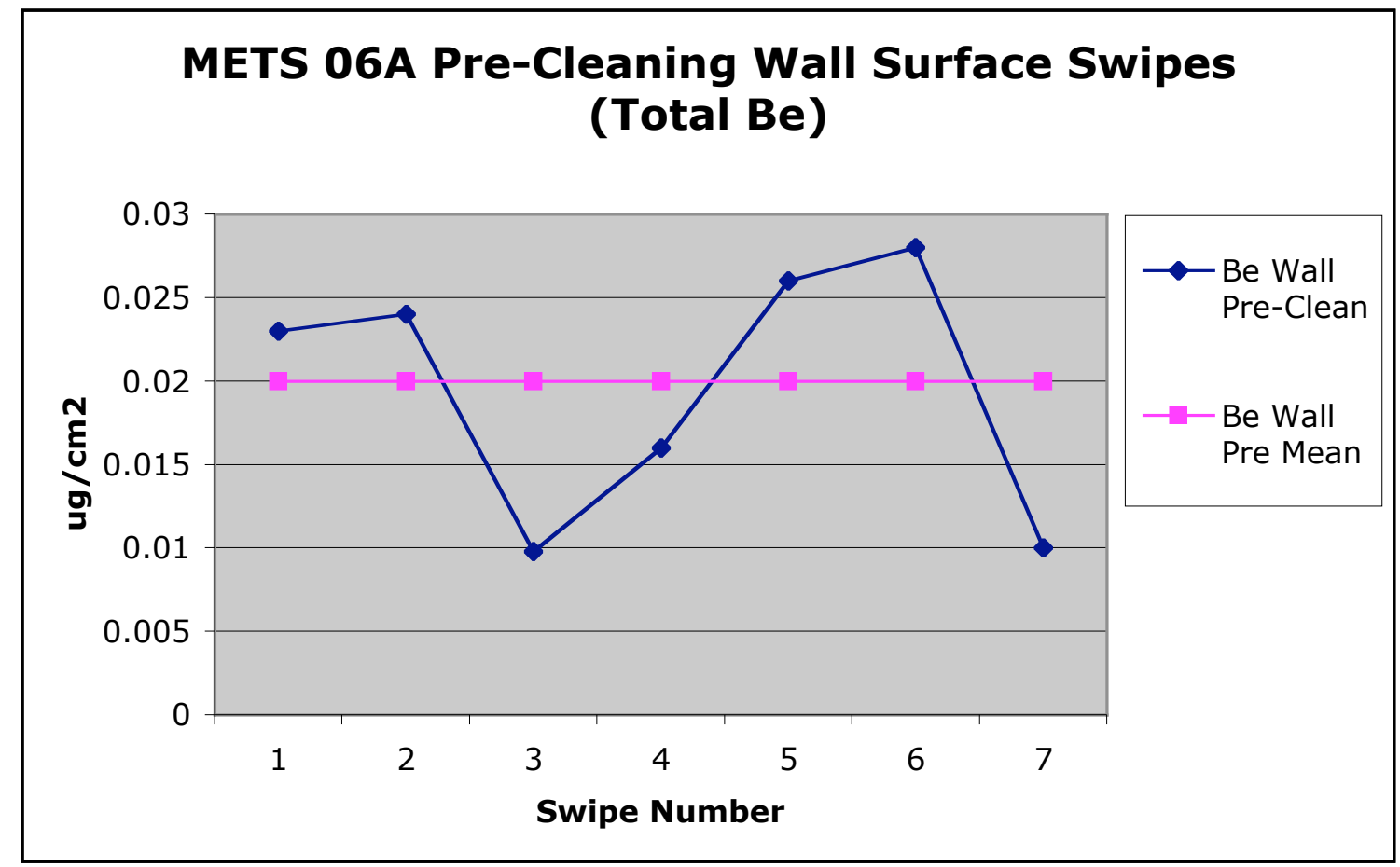


Table 14; Surface swipe results taken post-cleaning, from METS 04A, to provide a sideby-side comparison of wet versus dry swipe sampling protocol. From Batch \# 20080168 on $8 / 22 / 05$ are as follows:

\begin{tabular}{|c|c|c|}
\hline $\begin{array}{c}\text { Experimental Location } \\
\text { Sample \# }\end{array}$ & $\begin{array}{c}\text { Wet } \\
\mu \mathrm{g} / \mathrm{cm}^{2}\end{array}$ & $\begin{array}{c}\text { Dry } \\
\mu \mathrm{g} / \mathrm{cm}^{2}\end{array}$ \\
\hline $\begin{array}{c}\text { Post-Cleaning Firing Tank Door } \\
\text { FS\#2111505 -06 }\end{array}$ & 0.023 & 0.0012 \\
\hline $\begin{array}{c}\text { Post-Cleaning Firing Tank Door } \\
\text { FS\#2111507 -08 }\end{array}$ & 0.02 & 0.0017 \\
\hline $\begin{array}{c}\text { Post-Cleaning Firing Tank Floor } \\
\text { FS\#2111509-10 }\end{array}$ & 0.017 & 0.0017 \\
\hline $\begin{array}{c}\text { Post-Cleaning Firing Tank Floor } \\
\text { FS\#2111511 -12 }\end{array}$ & 0.015 & 0.0017 \\
\hline $\begin{array}{c}\text { Post-Cleaning Firing Tank Wall } \\
\text { FS\#2111513 - 14 }\end{array}$ & 0.012 & 0.0013 \\
\hline $\begin{array}{c}\text { Post-Cleaning Firing Tank Wall } \\
\text { FS\#2111515 - 16 }\end{array}$ & 0.013 & 0.0019 \\
\hline $\begin{array}{c}\text { Post-Cleaning Firing Tank Ceiling } \\
\text { FS\#2111517 - 18 }\end{array}$ & 0.018 & 0.0035 \\
\hline $\begin{array}{c}\text { Post-Cleaning Firing Tank Ceiling } \\
\text { FS\#2111519 - 20 }\end{array}$ & 0.039 & 0.0019 \\
\hline
\end{tabular}

Table 15; Surface swipe results taken post-cleaning subsequent to the entire METS series, these swipes taken after METS 06A, to provide a side-by-side comparison of wet versus dry swipe sampling protocol. From Batch \# 20080629 on 9/08/05 are as follows:

\begin{tabular}{|c|c|c|}
\hline $\begin{array}{c}\text { Experimental Location } \\
\text { Sample \# }\end{array}$ & $\begin{array}{c}\text { Wet } \\
\mu \mathrm{g} / \mathrm{cm}^{2}\end{array}$ & $\begin{array}{c}\text { Dry } \\
\mu \mathrm{g} / \mathrm{cm}^{2}\end{array}$ \\
\hline $\begin{array}{c}\text { Post-Cleaning Firing Tank Door } \\
\text { FS\#2111749 - 50 }\end{array}$ & 0.00073 & 0.00025 \\
\hline $\begin{array}{c}\text { Post-Cleaning Firing Tank Door } \\
\text { FS\#2111751 - 52 }\end{array}$ & 0.00025 & $<0.0002$ \\
\hline $\begin{array}{c}\text { Post-Cleaning Firing Tank Floor } \\
\text { FS\#2111753 - 54 }\end{array}$ & 0.001 & 0.00071 \\
\hline $\begin{array}{c}\text { Post-Cleaning Firing Tank Floor } \\
\text { FS\#2111755 - 56 }\end{array}$ & 0.002 & 0.0013 \\
\hline $\begin{array}{c}\text { Post-Cleaning Firing Tank Wall } \\
\text { FS\#2111757 - 58 }\end{array}$ & 0.00095 & 0.00049 \\
\hline $\begin{array}{c}\text { Post-Cleaning Firing Tank Wall } \\
\text { FS\#2111759 - 60 }\end{array}$ & 0.0014 & 0.00037 \\
\hline $\begin{array}{c}\text { Post-Cleaning Firing Tank Ceiling } \\
\text { FS\#2111761 - 62 }\end{array}$ & 0.00037 & 0.00024 \\
\hline $\begin{array}{c}\text { Post-Cleaning Firing Tank Ceiling } \\
\text { FS\#2111763 - 64 }\end{array}$ & 0.00041 & 0.0003 \\
\hline
\end{tabular}




\section{METS 06C Aluminum Powder System Comparison Test}

The final METS-related procedure performed as part of this research set was an attempt to perform an experimentally derived calibration of the METS sampling protocol. The comparison test procedures were applied to the post-detonation collection system, the cascade impactors, and the internal chamber environment. Parameters for this test were derived from the METS 04A mass and are compared to these results in the tables below.

Table 16; Collection levels immediately after the simulated METS 06C "detonation" of size-selected aluminum metal powder. METS 04A with side-by-side presentation below.

\begin{tabular}{|c|c|c|}
\hline $\begin{array}{c}\text { Sample Sequence } \\
\text { METS 06C Batch \# } \\
\text { METS 06C Sample \# }\end{array}$ & $\begin{array}{c}\text { METS 06C } \\
\text { Aluminum Metal } \\
\mathrm{mg} / \mathrm{m}^{3}\end{array}$ & $\begin{array}{c}\text { METS 04A } \\
\text { Total Beryllium } \\
\mathrm{mg} / \mathrm{m}^{3}\end{array}$ \\
\hline $\begin{array}{c}\text { Capture Tank } \boldsymbol{A} \\
\text { Batch\# 20082684 }\end{array}$ & $\begin{array}{c}552^{*} \\
\text { (15 seconds post- } \\
\text { fetonation) }\end{array}$ & $\begin{array}{c}\text { (30 seconds post- } \\
\text { detonation) }\end{array}$ \\
\hline $\begin{array}{c}\text { Capture Tank } \boldsymbol{B} \\
\text { Batch\# 20082684 }\end{array}$ & $\begin{array}{c}880 \\
\text { (Subsequent 2 } 1 / 2\end{array}$ \\
FS\#2112112 & $\begin{array}{c}\text { (Subsequent 5 minutes } \\
\text { post-detonation) }\end{array}$ \\
\hline
\end{tabular}

* +/- 25\% sampling system error should be considered per system's design parameters.

Table 17; Cascade impactor results for each stage after the simulated METS 06C "detonation" of size-selected aluminum metal powder. METS 04A (from Table 2) with side-by-side presentation below relating to METS 06C (Batch \# 20080607 \& 20080608, Field Sample \#s 2112114 - 122).

\begin{tabular}{|c|c|c|}
\hline $\begin{array}{c}\text { Cascade Impactor } \\
\begin{array}{c}\text { Size Fraction } \\
\text { (microns) }\end{array}\end{array}$ & $\begin{array}{c}\text { METS 06C } \\
\text { Aluminum Metal } \\
\mu \mathrm{g} / \mathrm{m}^{3}\end{array}$ & $\begin{array}{c}\text { METS 04A } \\
\text { Total Be } \\
\mu \mathrm{g} / \mathrm{m}^{3}\end{array}$ \\
\hline 10 to 9 & 460 & 28 \\
\hline 9 to 5.8 & 260 & 29 \\
\hline 5.8 to 4.7 & 210 & 23 \\
\hline 4.7 to 3.3 & 140 & 35 \\
\hline 3.3 to 2.1 & 64 & 44 \\
\hline 2.1 to 1.1 & 35 & 27 \\
\hline 1.1 to 0.65 & 18 & 0.77 \\
\hline 0.65 to 0.43 & 13 & 190 \\
\hline \hline Respirable Particulate Mass & & \\
$(<10)$ & 1200 & \\
\hline
\end{tabular}

Table 18; Internal firing tank air sampling, to replicate personal sampling parameters, for aluminum powder during METS 06C. METS 04A listed as side-by-side presentation below due to experimental similarities from Batch \# 20082691 (FS\# 2112124) as follows:

\begin{tabular}{|c|c|c|}
\hline METS Test & METS 06C & METS 04A \\
\hline $\begin{array}{c}\text { Homogenously Mixed Environment } \\
\text { (Standard Total Dust) }\end{array}$ & $\begin{array}{c}1100 \mu \mathrm{g} / \mathrm{m}^{3} \\
(25 \mathrm{minutes})\end{array}$ & $\begin{array}{c}41 \mu \mathrm{g} / \mathrm{m}^{3} \\
(106 \mathrm{minutes})\end{array}$ \\
\hline
\end{tabular}


Figures 11A\&B; METS 06C Tank A; Comparison Test with Aluminum at 40 microns.

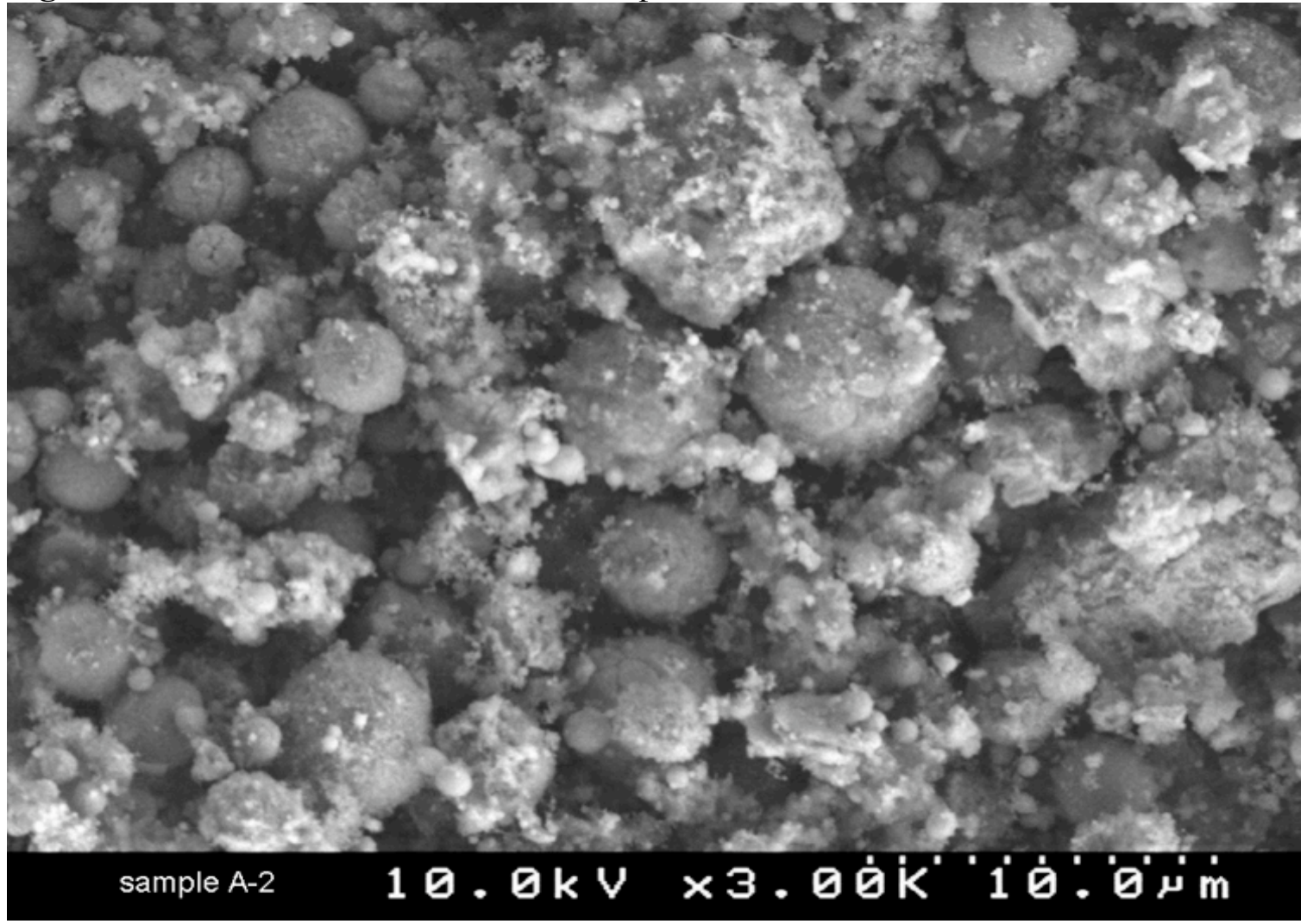

Label A: S300, filter A-2, 150u sq area, center of filter, 10KV

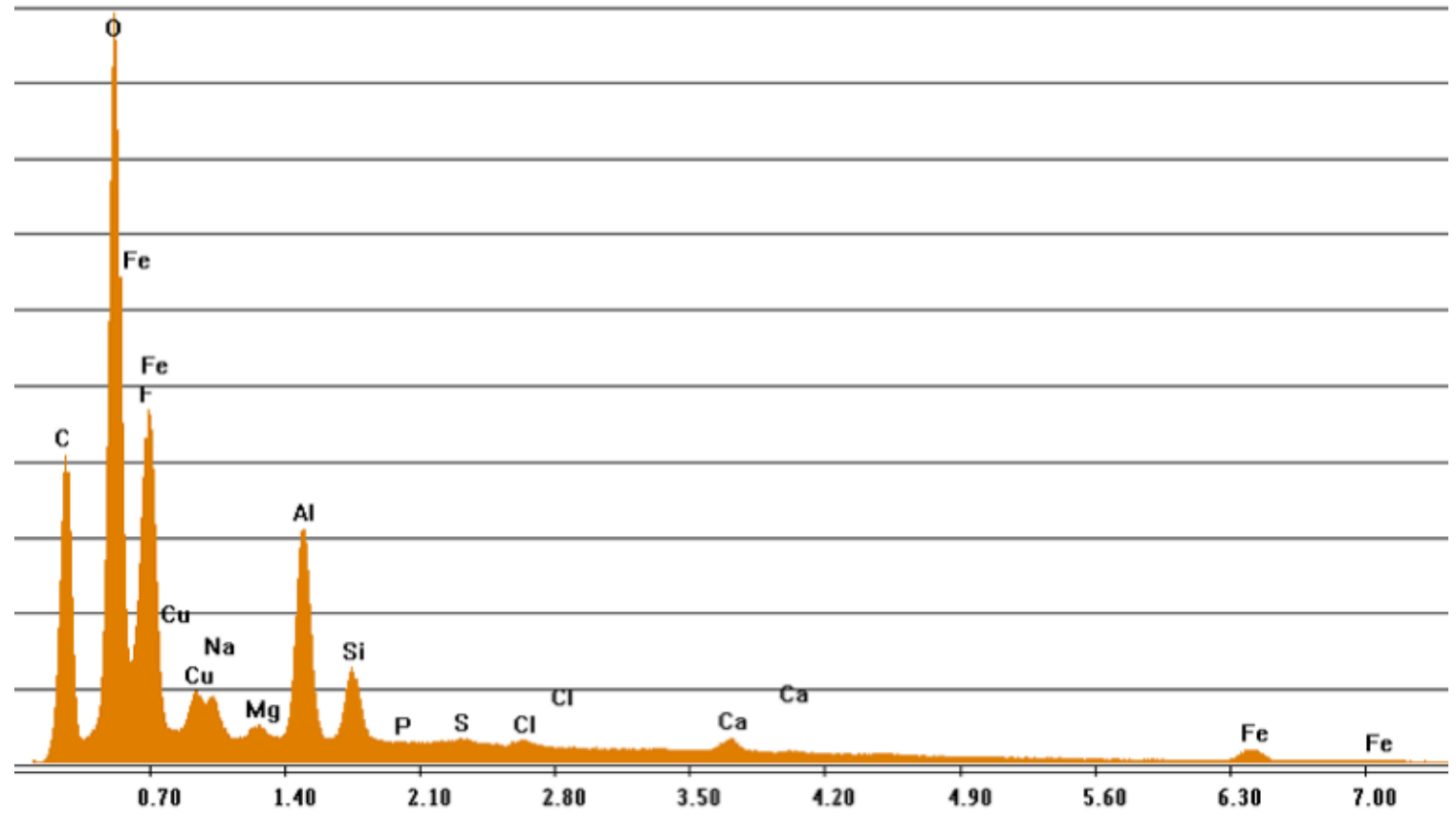


Figures 12A\&B; METS 06C Tank B; Comparison Test with Aluminum at $~ 80$ microns.

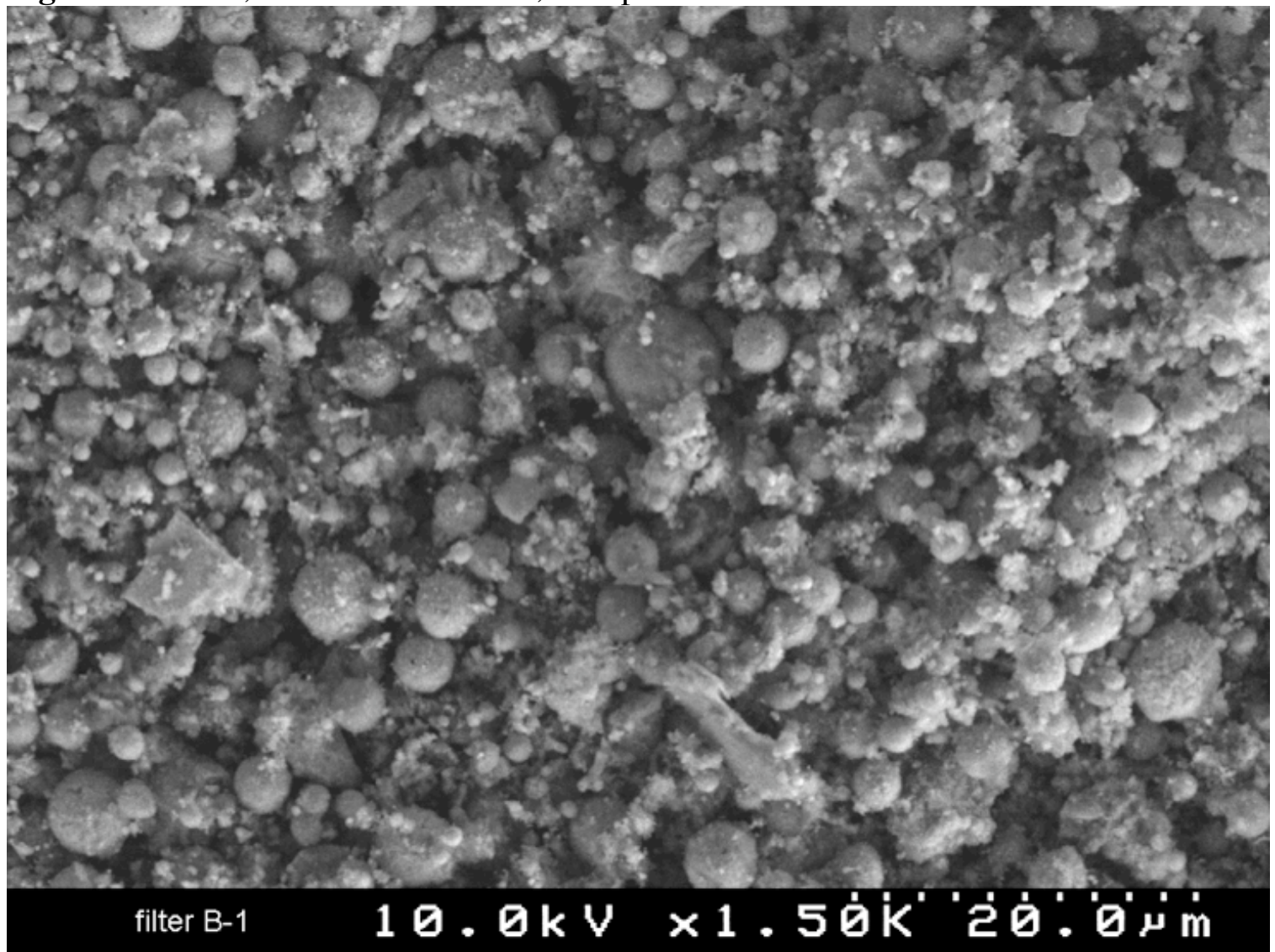

Label A: filter \#B-1, 200u sq surface area, 15KV

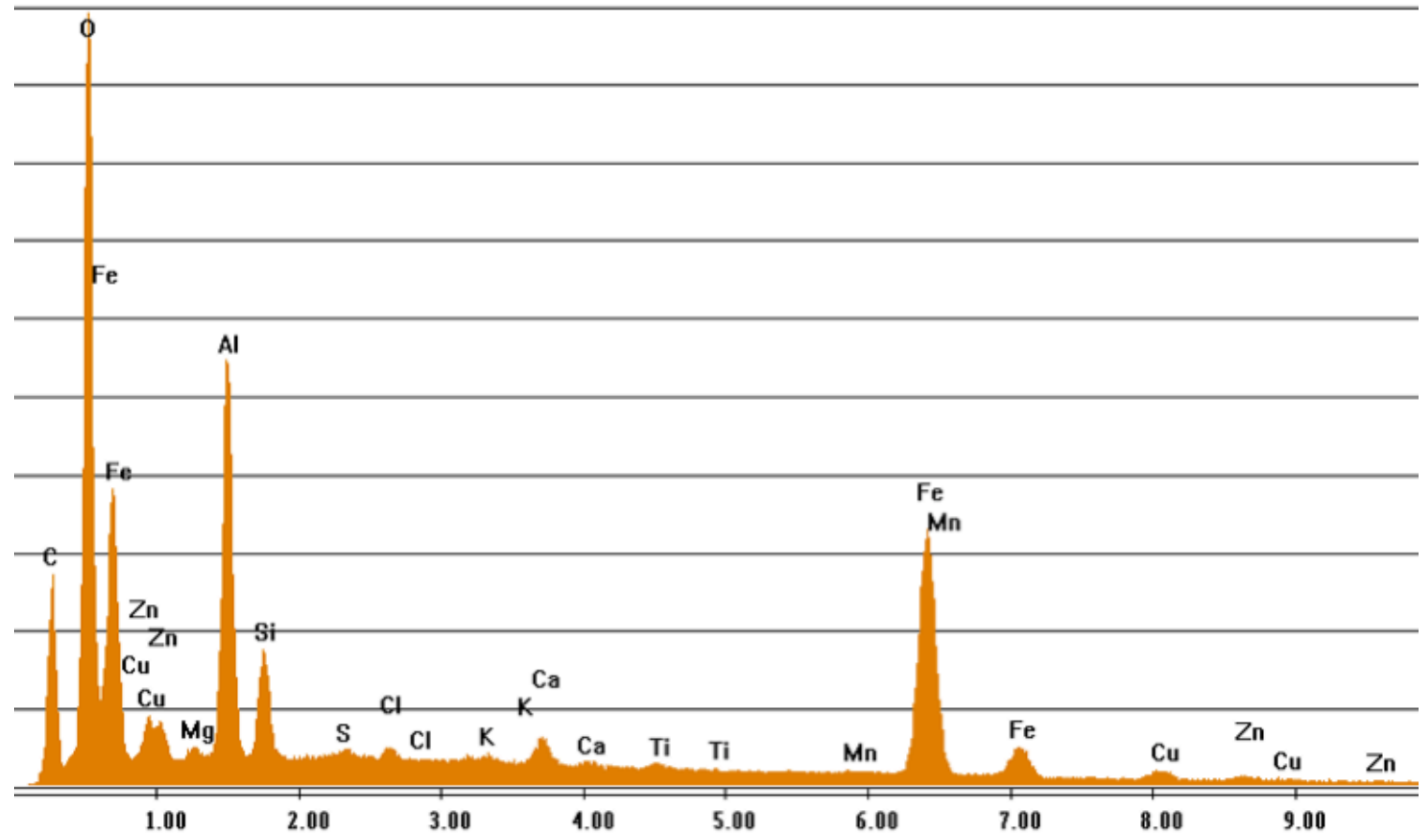




\section{DISCUSSION}

\section{Post-Detonation Collection System}

This initial experimental collection system was developed to narrow the capture window immediately post-detonation to evaluate the beryllium characteristics relating directly to dynamic event parameters within the firing tank's chamber. The first four-branch separator served to collect the 30 second (METS 04A) and 15 second (METS 05A and 06A) window immediately post-detonation to begin research into analogous sampling parameters for outdoor dynamic events. The second four-branch separator served to collect the subsequent four and one half minutes (METS 04A) and two and one half minutes (METS 05A and 06A) to serve as an airstream constituency most applicable to CFF fugitive emissions relating to chamber leakage in B801A historically. For each of the four-branch separator sampling cassettes, one was analyzed for total beryllium (beryllium metal and its oxide) and a replicate was analyzed for beryllium metal specifically.

The initial 30 second and 5 minute METS 04A collection time frames were determined to be visually too long a window due to the excessive build-up of particulate on the sampling cassette filters. This window was reduced to 15 seconds and $21 / 2$ minutes for the subsequent experiments (METS 05A and 06A) with this consideration in mind as the analytical results for METS 04A - 06A were not received until after the completion of the experimental set. Although the time window was narrowed to reduce particulate, the constant flow rates used throughout this research protocol, especially in the results from the primary Tank A, essentially renders this time differential mostly inconsequential.

\section{Beryllium Aerosolization Efficiency}

Exact beryllium mass and location parameters of these beryllium-containing dynamic event devices utilized in this METS experiment are not presented in this report due to classification reasons. However, it can be presented that both METS 04A and 05A contained the equivalent beryllium mass levels as was used in the METS 06C calibration test. A primary difference is that the METS $06 \mathrm{C}$ calibration protocol was entirely comprised of aerosolizable aluminum powder, which would be comparable to a complete vaporization of all beryllium material in METS 04A. The METS 06A dynamic device contained approximately 15 times less mass than its counterparts. The dynamic comparison of these experimental devices cannot be communicated in this paper due to classification issues (Please see classified version of this document). It can be said that METS 04A and 06A were designed in a similar manner; to transfer the highest level of dynamic forces to its beryllium component to simulate a worst-case scenario aerosolization potential. The design of METS 05A should be considered to transfer a moderate level of dynamic forces to its beryllium component in contrast to its counterparts.

The aerosolization efficiency is defined as quotient of the total beryllium analytical result masses (from Table 1) when compared between the METS dynamic devices in question. With its equivalent mass METS $04 \mathrm{~A}$ and $05 \mathrm{~A}$ can be directly compared for aerosolization efficiency. METS $04 \mathrm{~A}$ is therefore determined to have created $36 \%$ more 
aerosolized beryllium in the primary Tank A immediate post-detonation window when compared with METS 05A. Interestingly, the same comparison in the secondary Tank B subsequent post-detonation window, METS 04A is determined to have created $289 \%$ more aerosolizable beryllium than from METS 05A. As stated above, Tank B collection window parameters were derived from the constituency most likely to be linked to CFF chamber fugitive emissions into the B801A ADHZ and common areas, a significant difference between the dynamic event potential outcomes relating to these device designs.

The results from Table 1, with the design differences discussed above, can therefore be rendered equivalent within and between these METS devices by multiplying the METS 06A results (Table 1) by 15, the mass differential to equalize the parameters that differentiate its design from METS 04A and 05A. This process is reflected in Figure 13 below which is adapted from Figure 6 parameters. Utilizing these equivalent masses, it can be seen that based on the analytical results, METS 06A appears to have the most

Figure 13; Collection comparisons with the devices rendered comparable in initial beryllium mass with Table 1 results for METS 06A multiplied by 15 (mass differential).

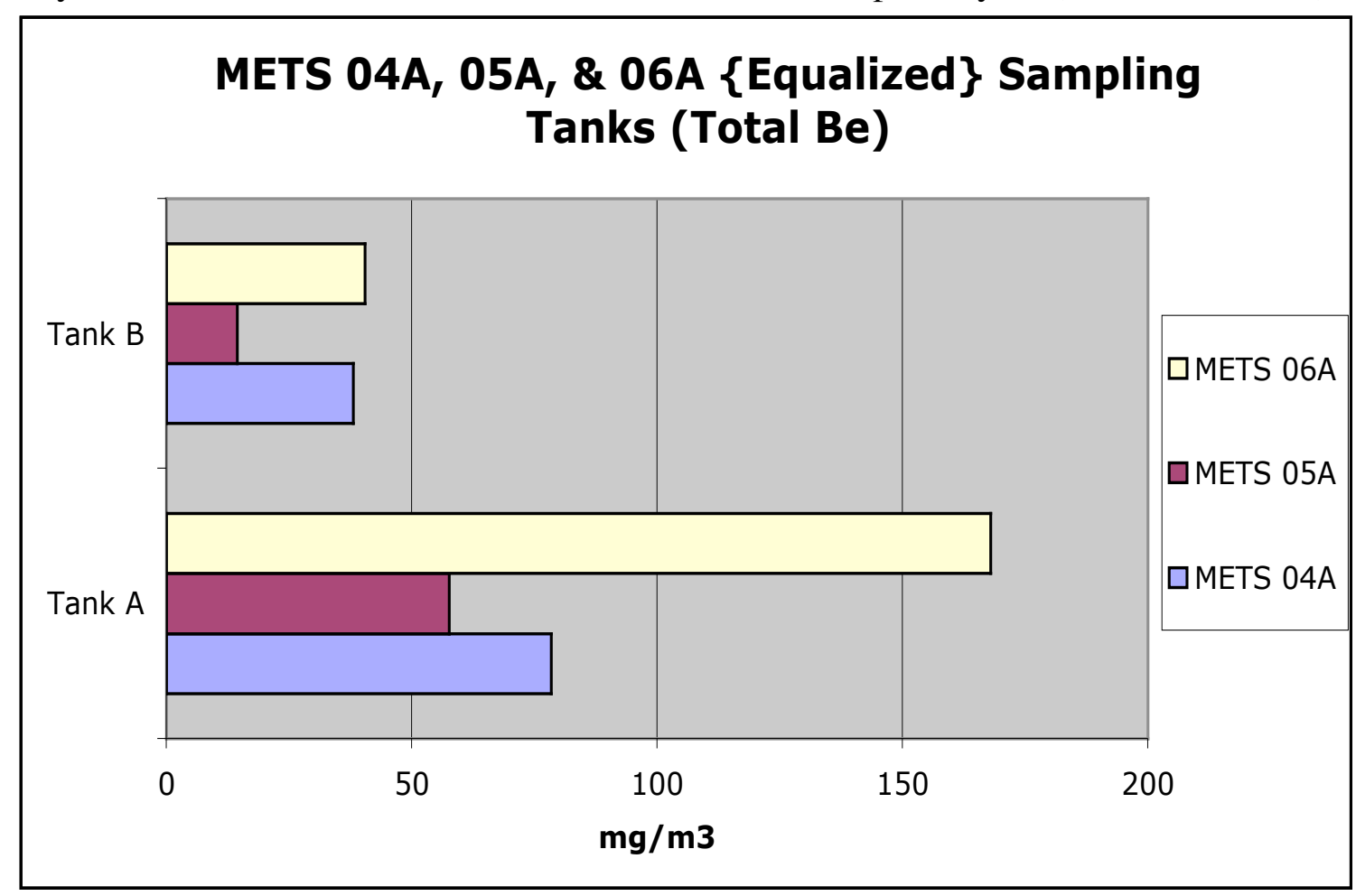

dynamic effect on its beryllium components. Looking at the Tank A immediate postdetonation window, METS 06A dynamic design is $214 \%$ more effective in generating aerosolizable beryllium than METS 04A and 292\% more than METS 05A. The Tank B collection window renders METS 06A and 04A much more comparable, or essentially equal with collection error considerations, with METS 06A merely 6\% more efficient METS 06A. Tank B comparisons remain weighted heavily toward METS 06A when compared to METS $05 \mathrm{~A}$ with a $281 \%$ greater beryllium aerosolization efficiency. 
Beryllium Oxide Production

For the information presented in Table 1 for direct comparison of METS 04A and 05A, the differential in total beryllium to beryllium metal is essentially equal. There are even indications that more beryllium metal is produced in METS 04A, however these nearly equivalent levels are a better indication of the uniformity of the four-branch airstream separator than anything else.

Four-Branch Airstream Separator Error

Of the four cassettes available for each of the Tank A and Tank B collection opportunities, the samples sent in for either total beryllium or beryllium metal analysis are entirely random as they are numbered for sequence in series. With the assumption that no beryllium oxide is produced (of measurable mass quantities, therefore negating the $<20 \mathrm{~nm}$ particulate population seen on the SEM images discussed below) the differential between the duplicate samples for METS 04A and 05A range from 0 to $9 \%$ throughout the results in Table 1. Although this differential should be a focus in future experimental efforts with this collection system, a rough estimate of $+/-10 \%$ airstream uniformity among the four duplicate samples can be attributed to the four-branch airstream separator.

\section{Collection Method Error}

The analytical results listed in Table 1 are results received. However, due to the nature of the collection system's connection to vacuum tanks and not to an ambient air environment, a sampling system error has been derived so it can be applied throughout this table. The initial mass flow controller is listed for an accuracy reading of $1.5 \%$ with an oulet pressure certified to 23 inches mercury gauge (" $\mathrm{Hg}$ g). This flow controller is placed in series with a rotameter that is certified for accuracy at $+/-5 \%$. Therefore, it is a conservative assumption that the system should have a total of $+/-10 \%$ throughout, although it is most applicable to the information presented in Table 1 and Figure 6 . This error should be uniform throughout due to consistency in application throughout all 3 experimental collection opportunities however due to the system's design parameters it can be applied throughout the test series.

\section{Scanning Electron Microscopy}

With rare exceptions, all of the material on the filters (Figures 7A\&B - $10 \mathrm{~A} \& \mathrm{~B}$ ) has a spherical morphology. It is not clear how much of the tight clumping of particles is a result of the encapsulant that thoroughly coated all of the samples. I suspect that the overall structure would be a looser, open network of particles in the uncoated state.

\section{Morphology}

Filter deposit is made up entirely of spherical particles ranging in size from $>5.0 \mu \mathrm{m}$ to $<20 \mathrm{~nm}$ diameter. Working with random $10.0 \mu \mathrm{m}^{2}$ areas on each filter the following statistical estimates were obtained:

\section{Spherical particulate}

- $5 \%$ of the particles are $1.0 \mu \mathrm{m}$ or larger diameter. 
- $<0.5 \%$ of the particles were larger than $5.0 \mu \mathrm{m}$ diameter.

- $10 \%$ of the particles are in the range $<1.0 \mu \mathrm{m}$ to $>0.5 \mu \mathrm{m}$ diameter.

- Particles $<0.5 \mu \mathrm{m}$ diameter make up the remaining bulk of filter sample deposits.

- Most larger particles, $>0.2 \mu \mathrm{m}$ diameter, were observed to be covered with a finer dispersion of particles.

- Particles <20.0 nm diameter could be resolved throughout all filter samples.

\section{Non-spherical particulate}

- Rare, less than one per $100 \mu \mathrm{m}^{2}$ area.

- Non-spherical particulates are typically $>5.0 \mu \mathrm{m}$ size.

- Typical non-spherical particulate composition either iron $(\mathrm{Fe})$ or silica $(\mathrm{Si})$.

\section{Chemical Composition}

Aluminum ( $\mathrm{Al})$ and Iron $(\mathrm{Fe})$, along with a significant percentage of oxygen $(\mathrm{O})$ are present in all EDX scans from large surface areas to individual particles, suggesting $\mathrm{Al}$ and Fe oxides are present. Due to the X-ray source for the SEM equipment, beryllium cannot be speciated directly and is not presented in the information that follows. The overall weight ratio, expressed as a percentage of particulates observed, is very roughly 1:1 for $\mathrm{Al}$ and $\mathrm{Fe}$. Copper $(\mathrm{Cu})$ and zinc $(\mathrm{Zn})$ are present in all scans each representing approximately 1 to 5 percent by weight of the overall bulk. Sodium $(\mathrm{Na})$, magnesium $(\mathrm{Mg}), \mathrm{Si}$, sulfur $(\mathrm{S})$, chlorine $(\mathrm{Cl})$, calcium $(\mathrm{Ca})$, and manganese $(\mathrm{Mn})$ were variously detectable and collectively represent perhaps $<5.0$ percentage by weight (wt\%) of the bulk. An overall composition is roughly as follows:

- $\mathrm{Al}$ oxide $>45 \mathrm{wt} \%$

- Fe oxide $>45 \mathrm{wt} \%$

- $\mathrm{Cu}$ (oxide) $<5 \mathrm{wt} \%$

- $\mathrm{Zn}$ (oxide) $<5 \mathrm{wt} \%$

- $\mathrm{Na}, \mathrm{Mg}, \mathrm{Si}, \mathrm{S}, \mathrm{Cl}, \mathrm{Ca}$ and Mn collectively $<5 \mathrm{wt} \%$

A specific break down of the chemical composition of the three METS 04A and 05A samples analyzed (Figures $7 \mathrm{~A} \& \mathrm{~B}-10 \mathrm{~A} \& \mathrm{~B}$ ) are additionally presented. All three samples were essentially comparable with sample B1 (METS 05A Tank A from Figures $9 \mathrm{~A} \& \mathrm{~B}$ and $10 \mathrm{~A} \& \mathrm{~B}$ ) perhaps containing more $\mathrm{Al}$ than $\mathrm{Fe}$, but it was difficult to come up with a reliable ratio. An observed, approximated ratio of 3:2 Al to Fe is present in some fields, however, some scans were closer to the 1:1 ratio observed in A1 (METS 04A Tank A from Figure 7A\&B) and A2 (METS 04 Tank B from Figure 8A\&B). Due to the particulate nature of the samples, and variations in concentrations and bulk density, it is extremely difficult to come up with quantitative numbers. After a thorough review of the overall results provided, cumulatively the concentrations presented as part of this SEM analysis are reasonably representative of the bulk deposits. As noted above, the beryllium particulates could not be identified, thus are not included in the analysis. Carbon was also ignored as it is the primary constituent of the encapsulation material that coated all filter material. It is possible that some of the very fine particulate matter, $<20 \mathrm{~nm}$ diameter, contains beryllium, but no particulates comparable in size to the spherical oxide 
particles were found to be suggestive of beryllium within the limitations presented by the EDX analysis.

\section{$\underline{\text { SEM Analysis }}$}

The viewing of the SEM photos assists in the delineation of the composition of particulate size populations when comparing the Tank A immediate post-detonation window and the Tank B subsequent window. The vast majority of the filter area, screened during SEM analysis, is seen to be populated by particulate $<0.5 \mu \mathrm{m}$ with a substantial concentration of particulate at and below the $20 \mathrm{~nm}$ in the airstream captured in Tank A is best seen in the higher magnification levels from Figure 10A. As the temperatures and pressures within the firing chamber begin to cool, these nanoparticulate begin to agglomerate to form larger, hollow spheres (Figure 14). The hollowness of the spheres, as well as their being comprised of this much smaller particulate, was seen in METS 03 (Figure 15) with much larger particulates collected as bulks prior to cleaning of the firing tank, then on the B850 firing table.

Figure 14; Creation of hollow spheres, seen here in the Tank B second post-detonation window, formed by the agglomeration of $<0.5 \mu \mathrm{m}-20 \mathrm{~nm}$ particulate that are the majority of the Tank A content.

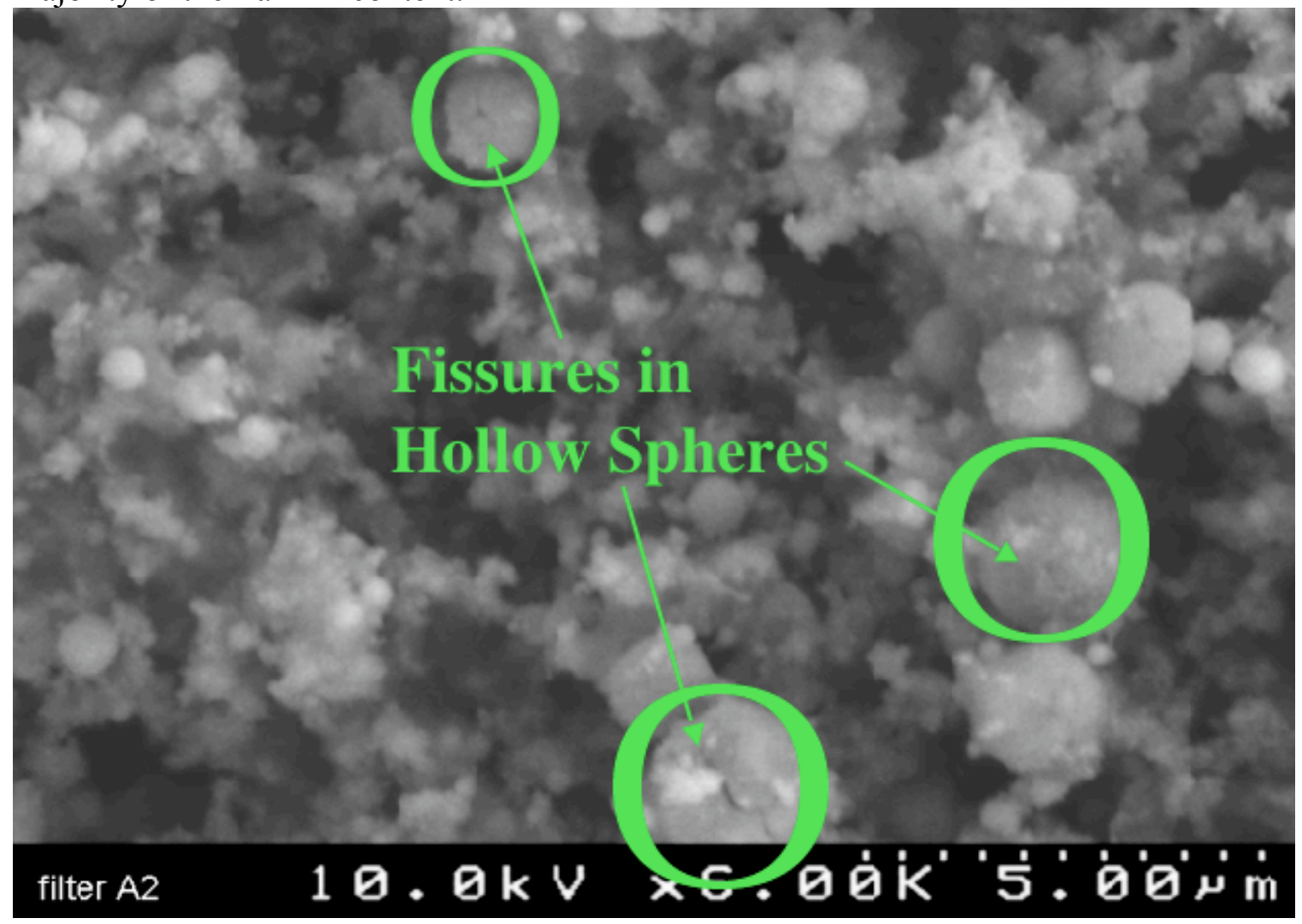

The creation of such a high concentration of nano-particulate has an interesting outcome in that the overall agglomeration of the relatively much larger particulate as seen in Figure 15 does not seem to be present in this METS series. It can be presumed that the 
highly dynamic design of these experimental devices creates this significant quantity of nano-particulate and, in the absence of intervening factors, will create a high population of respirable particulate during the cleaning phases. As seen below, it should be predictive that this will also lead to elevated personal exposure potential as the low density of these small particulate increase both the ease of reaerosolization and the amount of time that these particulates remain airborne increases inversely to the size of a given particulate, in line with the terminal settling velocity parameters:

$$
\begin{gathered}
\mathrm{V}_{\mathrm{TS}}=\left(\rho_{P}\right)\left(d^{2}\right)(g) / 18 \eta \text { for } d>1 \mu \mathrm{m}, \\
\mathrm{V}_{\mathrm{TS}}=\text { Terminal Settling Velocity }(\mathrm{cm} / \mathrm{s}) \\
\rho_{P}=\text { Density of the Particle }\left(\mathrm{g} / \mathrm{cm}^{3}\right) \\
d=\text { Diameter of Sphere }(\mathrm{cm}) \\
\mathrm{g}=\text { Acceleration of Gravity }\left(\mathrm{cm} / \mathrm{s}^{2}\right) \\
\eta=\text { Gas Viscosity }(\mathrm{g} / \mathrm{cm}-\mathrm{s})
\end{gathered}
$$

Valid only for unit-density spheres at standard conditions;

$$
\mathrm{V}_{\mathrm{TS}} \approx 0.003\left(d^{2}\right) \quad \text { for } 1<d<100 \mu \mathrm{m}
$$

Therefore, as overall particulate size trends toward $1 \mu \mathrm{m}$ and smaller the particulates will have a tendency to not settle. This is a difficult variable to ensure reduced and controlled personal exposure potential during re-entry and subsequent chamber cleaning procedures.

Figure 15; SEM picture from METS 03 with larger, yet similarly agglomerated particulates and indications that they are hollow as seen in Figure 14 above.

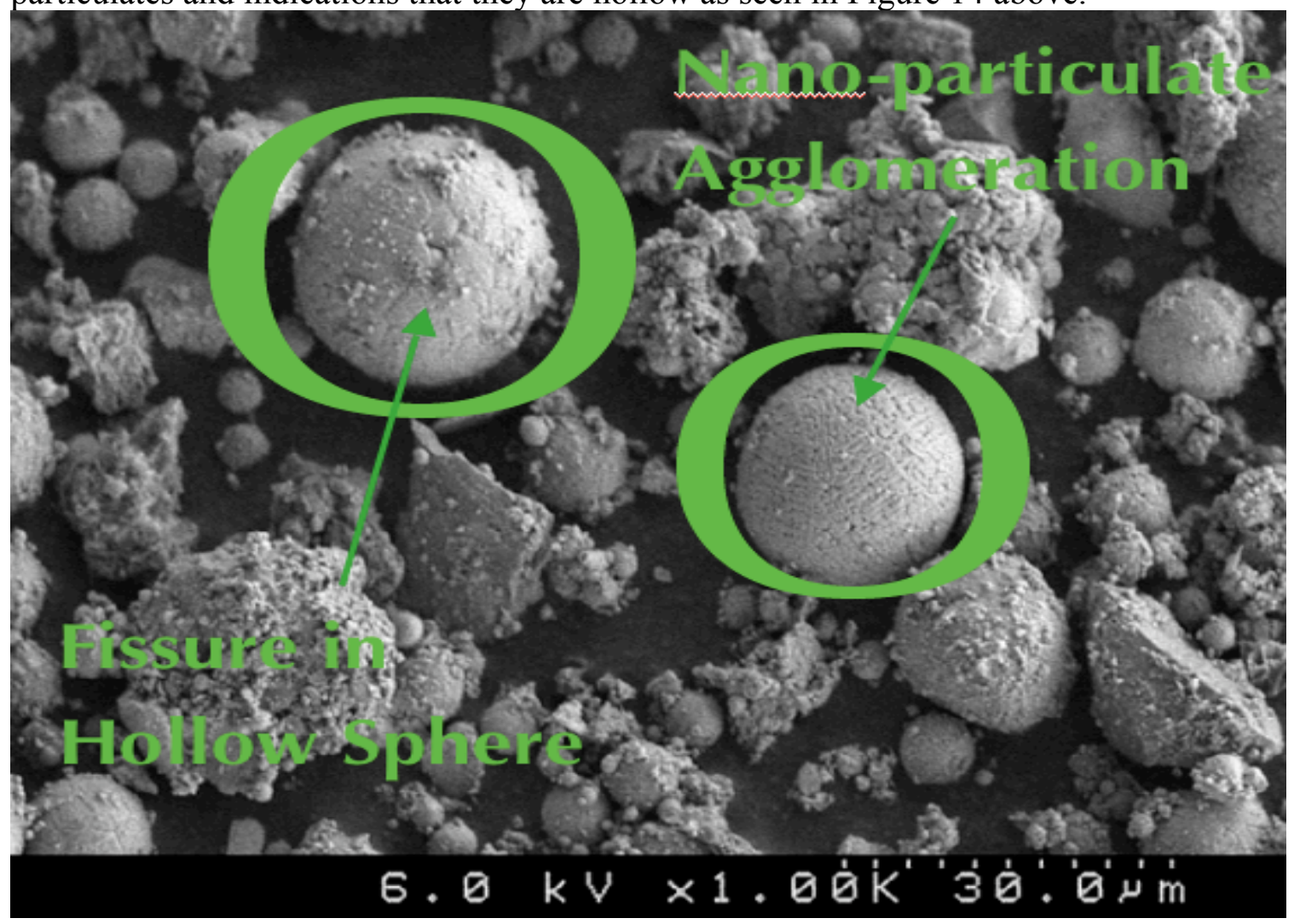


METS 06C Calibration Test

The composition of material from METS 06C (Figures 11A\&B and 12A\&B) fits very well with the expected overall levels of the aluminum powder utilized in this experiment. Additionally, the size-selective parameters indicate a greater concentration of particulate $>5.0 \mu \mathrm{m}$ size and a similar count of particulate $<1.0 \mu \mathrm{m}$ size, absent the $<20 \mathrm{~nm}$ particles.

\section{Cascade Impactors}

Duplicate cascade impactors were taken simultaneously in a homogenously mixed internal tank environment, placed on the closed firing tank floor's surface for a specified period of time. The mixing system and the cascade impactors ran for 5 minutes during METS 04A and both ran for 20 minutes during METS 05A and 06A. The added running times for METS 05A and 06A were put into place, when compared to METS 04A, due to the dynamic design differences versus METS 05A and the substantially lower beryllium mass in METS 06A. The extended sampling periods were to assure a lower detection limit for both of these experiments, but especially focusing on METS 06A.

\section{Respirable Beryllium Mass}

The dynamic event that was incorporated into the design of METS 04A is most apparent in light of the cascade impactor's analytical results. Even if the masses of METS 06A and 04A were equilibrated, METS 04A produced over 11 times more respirable mass in the cascade impactors within the experimental parameters of this research. This three orders of magnitude difference is not fully understand when compared to the postdetonation collection system results as the Tank B parameters are heading toward the final equilibrium particulate constituency that becomes evident as the firing tank's internal environment approaches background and particulate settling occurs. The high beryllium mass quantities in the $1-10$ micron range for METS 04A do indicate that as the post-detonation environment begins approaching background levels and reach an ambient equilibrium, there is a substantial increase in particle size when compared to the SEM results. This agglomeration that occurs in this process significantly increases the overall size of the particulate size fraction that will be present at the time of firing tank, or CFF chamber, initial re-entry and cleaning procedures.

Beryllium Oxide Production

METS 06A produced 17\% more total beryllium respirable fraction mass than the beryllium metal designated cascade impactor. This additional amount can be directly attributed to the creation of beryllium oxide as a by-product of the METS 06A dynamic event. METS 04A produced essentially identical total beryllium and beryllium metal results which indicate that the creation of beryllium oxide was not a factor within the confines of the firing tank. This information should be taken with a caveat in that the smallest quantifiable size fraction ( 0.65 to 0.43 microns) produced approximately $50 \%$ more mass as beryllium oxide. Taken with the SEM results, with such a high concentration of $<20.0 \mathrm{~nm}$ particulate formation (Figure 7A) and the truly significant increase in available surface area to mass associated with sub-micron (or quasi nano-) particulate, the toxicologically available beryllium oxide surface area may be orders of magnitude greater with a METS 04A design. Unfortunately this comparison cannot be 
made with the METS 06A design as the results in the smallest size fractions are below the limit of detection, even with the increased sampling time.

\section{Chamber Environment Personal Air and Surrogates}

After a minimum of 24 hours post-detonation the firing tank door is opened for the first time after the particulate has been given the same opportunity to settle as prescribed within the CFF chamber procedures. Placed inside the firing tank at this time, along with the duplicate cascade impactors, are additional personal air sampling devices that serve as personal exposure potential surrogoates. These sampling devices include traditional total dust collection methods as well as experimental evaluation of various inhalable and respirable size-selective collection devices available for a couple of experimental testing opportunities during METS 05 and 06. All samples were taken simultaneously in a homogenously mixed internal tank environment. After settling, the firing tank door is opened again and personal sampling devices were turned off and removed. It is at this time that the procedures for the low-pressure washing, HEPA vacuuming, and initial cleaning of the internal firing tank are performed. These employees, wearing appropriate protective gear, don the required personal sampling pumps to document their true personal exposures and reflect the potential for exposure for those performing similar work. Although these workers remain outside the firing tank at all times and do not enter the firing tank prior to its full cleaning, the personal exposure levels are expected to be a rough estimate due to the confined nature of the surfaces cleaned.

\section{Personal Air Sampling}

When comparing true personal air sampling results associated with the firing tank procedures requires focusing on the actual monitoring result and not the derived 8-hour time weighted average that is required for personnel monitoring. An additional focus should also be placed on the specific firing tank procedures performed: sample taking, sampling device retrieval, equipment removal, firing tank cleaning, and shot set-up. Each of these procedures are derived from, and are comparable to, CFF chamber activities. The biggest difference between these METS-related personal sample results and those that occur within CFF is that the workers are standing directly outside of the METS firing tank in fresh air. CFF personnel are not afforded this luxury and trending of elevated personal samples taken from B801A activities within the chamber indicate that there is a uniform exposure potential for all workers when contamination is re-aerosolized. Some of these METS operative parameters are similar in that all workers are standing together immediately outside the firing tank entrance, so that the re-aerosolized material can be somewhat uniformly distributed should contamination be disturbed either by procedural efforts or by the occasional shifting of winds seen on and around the B812 firing table.

METS 04A's combination of beryllium mass and dynamic efficiency yielded the highest personal exposure results of the entire test series. These eight personal sampling results were all above the limit of detection with a range of 0.051 to $0.61 \mu \mathrm{g} / \mathrm{m}^{3}$ over the brief monitoring periods that are typically associated with the minimal surface area to clean and work with in the METS firing tank. These sample results should be directly compared to the Beryllium Action Level of $0.2 \mu \mathrm{g} / \mathrm{m}^{3}$ when developing decision-making parameters for equivalent dynamic events in the CFF chamber. For METS 04A, five of 
the eight sample results are above this Action Level. For comparison sake, only two of the ten METS 05A personal sampling results are above the limit of detection and both of those were below the Action Level. None of the seven personal sampling results from METS 06A were above the limit of detection, as were the two samples relating to shot set-up procedures.

Chamber Environment Personal Surrogates

Internal firing tank total dust and size-selective air sampling, to replicate personal sampling parameters, for Beryllium Metal during METS 04A - 06A were all taken at the same time and are presented in Tables 10 and 11. The total dust cassettes used in the sampling parameters in Table 10 indicate results that share similarities with the cascade impactor results. The METS 04A result of $41 \mu \mathrm{g} / \mathrm{m}^{3}$ is almost three times higher than the METS 06A result equilibrated for mass. Additionally, the METS 04A result is 21 times higher than the METS 05A homogeneously mixed internal tank environment. All of these total dust samples were taken with personal sampling pumps at the standardized 3.0 1/min flow rate and at personnel breathing zone levels. It should therefore be considered indicative, if not predictive, of the true personal sampling results discussed above which also reflected an order of magnitude increase in exposure potential during METS 04A procedures when compared to METS 05A and its device's equivalent beryllium mass level.

\section{Chamber Environment Size-Selective Devices}

The experimental application of size-selective personal sampling devices was performed in the future anticipation of a change in the occupational exposure limit monitoring parameters for beryllium which currently require a $37 \mathrm{~mm}$ total dust cassette. As these sampling devices are size-selective, they cannot be compared to the existing Beryllium Action Limit or the Permissible Exposure Limit as these are based on standard total dust cassettes which are not size-selective by design. It is predicted that future occupational exposure limits for beryllium will no longer require total dust cassettes, a truly archaic application. Without size-selective, particle count, and/or surface area ratios that are sought in this experimental effort, one cannot correspond directly to available beryllium toxicological or epidemiological beryllium (Tinkle et al 2003, Eisenbud 1998, Kriess et all 1997).

Typical comparisons between total dust and inhalable ( $<100$ micron) are often difficult to derive and should be considered operationally and procedurally dependent (Martin and Zalk, 1998). It is generally recognized that respirable ( $<10$ micron) sampling methods will collect less mass than total dust and total dust to inhalable ratios can range from $1: 1$ to $3: 1$ or greater. As a general rule when attempting to compare these personal sampling exposure potential parameters it is essential to focus on the energy in the system that is producing the aerosolizable particulate such that the higher the energy the smaller the average particulate generated. It is believed by the authors of this report that this METS research is indicative of the highest of energies to be produced in a closed system. A byproduct of this is that this beryllium material's particulate size should be primarily in the vapor stage as initially produced. For size-selective parameters this means that there should not be much of a difference, if any, between total dust and inhalable methods 
especially and between total dust and respirable particularly. This parable has held mostly true with these devices under the existing experimental parameters.

The variations between all the different samplers are quite high especially when considering that the experimental parameters between all of them were alike. It is for this reason that this additional experimental procedure was requested by this METS research team from NIOSH. The NIOSH research is seeking a comparison of sampling collection results under like conditions in light of pending occupational exposure limits for beryllium from ACGIH. For the sake of this METS protocol it was useful to put these personal sampling devices into practice for consideration by the Site 300 Industrial Hygienist for potential use at Site 300 and within CFF particularly. In this regard, the IOM sampler has stood out as an outstanding personal monitoring device. It's ease of use an assembly belittles the complexity in its simplified design. This single personal sampling item can obtain simultaneous inhalable and respirable mass concentrations from within the same lightweight device. The duplication opportunities in presented in Table 11 for the IOM sampler during METS 06A yielded roughly comparable levels for both the respirable and the inhalable size fraction results. The differential in the results between METS 05A and 06A (Tables 10 and 11) indicate roughly comparable sample result ratios between each of the total dust, inhalable, and respirable monitoring opportunities.

\section{Surface Swipe Sampling}

Surface swipe sampling was performed on the firing tank's surfaces to indicate representative surface levels for total beryllium. Table 12 and Figures $11-14$ are all taken as wet surface swipes and are indicative of the surface levels of the firing tank's interior surfaces immediately prior to the initial cleaning of the tank's interior. These wet surface swipes were taken after a minimum of 24 hours after METS 05A and 06A detonation and after the settling of the tank's internal environment subsequent to the removal of sampling devices relating to the monitoring protocol during the chamber's homogenous mixing. The means of these results are indicative to the 15 times higher mass in METS 05A when compared to 06A, with the METS 05A mean remaining 21/2 times higher when equalized for beryllium mass. This level is roughly comparable to the total dust area air sample differential, giving an interesting link between the device masses and the air/surface interaction. 
Wet vs. Dry A comparison of wet swiping to dry swiping protocol are presented in Tables $13-15$ and were taken post-shot and prior to cleaning, as well as after tank cleaning procedures. The means derived from these tables are presented for comparison below.

Table 19; Comparison of the means derived for the results in Tables 13 - 15:

\begin{tabular}{|c|c|c|}
\hline $\begin{array}{c}\text { Surface Swipe Test } \\
\text { Parameters }\end{array}$ & WET & DRY. \\
\hline $\begin{array}{c}\text { METS 04A } \\
\text { Pre-Cleaning }\end{array}$ & $0.48 \mu \mathrm{g} / \mathrm{cm}^{2}$ & $0.018 \mu \mathrm{g} / \mathrm{m}^{3}$ \\
\hline $\begin{array}{c}\text { METS 04A } \\
\text { Initial Post-Cleaning }\end{array}$ & $0.020 \mu \mathrm{g} / \mathrm{cm}^{2}$ & $0.0018 \mu \mathrm{g} / \mathrm{m}^{3}$ \\
\hline $\begin{array}{c}\text { METS 06A } \\
\text { Completed Post-Cleaning }\end{array}$ & $0.00089 \mu \mathrm{g} / \mathrm{cm}^{2}$ & $0.00048 \mu \mathrm{g} / \mathrm{m}^{3}$ \\
\hline
\end{tabular}

With the results presented in Table 19 there may be a number of conclusions that can be drawn. First and foremost, when wet swipes are taken on dirty and/or contaminated surfaces they will invariably collect much more mass. There is an order of magnitude difference between wet and dry samples, whether taken before cleaning begins or after a gross initial wet cleaning is performed, even if the gross cleaning reduced surface beryllium levels an order of magnitude. A far more comparable set for both METS and CFF chamber parameters can be seen after the completion of all post-shot cleaning protocol. This is seen after METS 06A procedures where the means are merely a 2 to 1 differential from wet to dry surface swipe protocol. It should be noted that the final encapsulation step that occurs after the completed cleaning of CFF chamber surfaces was not applied to the METS firing tank results presented in Tables $13-15$ and 19.

\section{CONCLUSIONS}

The purpose of this work was to examine the environmental, safety, health and operational aspects of detonating a confined explosive test apparatus that has been designed to maximize the dynamics of impact on beryllium metal components for Contained Firing Facility (CFF) applications. The combination of experimental collection and evaluation methods designed and implemented for this research provided an excellent evaluation of immediately post-detonation by-products reflecting a potential WCS beryllium aerosolization explosive event. The explosive devices were appropriately designed to serve as a scaled model for the dedicated METS firing tank and the CFF chamber. The experimental results provided appropriate information to develop operational parameters necessary for conducting full-scale beryllium-containing experimental tests with similar designs within CFF and B801A.

\section{Research Goals}

The METS $04-06$ proposal sought to address these requirements and detection parameters to best mitigate this component for the B801 complex and its environmental 
and personnel exposure concerns. A research protocol for the detection, collection, and characterization of aerosolized beryllium particulates, both immediately post-detonation and during worker clean-up procedures, was developed to assist in the determination of the appropriate response for full-scale dynamic test parameters to control the experimental by-products, predict fugitive emissions and chamber purging constituency. In addition, practically it is necessary to learn how to preventatively control beryllium personal exposure potential while cleaning the CFF with properly designated personal protection equipment to prepare the chamber for timely re-use. In line with our research specific goals specified during this METS proposal, the following goals were achieved:

- The cumulative collection and analysis system developed by this METS research team succeeded in achieving an understanding of the airstream constituency related to the leak potential within B801A ADHZ and common areas resulting from worst-case scenario (WCS) dynamic events with a beryllium-containing device.

- The particle size distribution relating to three distinct post-detonation windows was determined and can be compared and contrasted across the three experimental designs.

- Although the beryllium metal and beryllium oxide content and size distribution both immediately post-detonation and during clean-up procedures was monitored for, the overall limited beryllium oxide production rendered results within the range of sampling equipment error and of reduced value.

- Consistent across experimental designs was the beryllium morphology information. The SEM analysis indicates that the immediate post-detonation window is made up entirely of spherical particles ranging in size from $>5.0$ $\mu \mathrm{m}$ to $<20 \mathrm{~nm}$ diameter. These experimentally derived results answer previously conflicting expert advice, now indicating we should be using vaporization mechanics, and not fractionalization, when working with these WCS dynamic designs in future post-detonation science efforts.

- The collective analytical information indicates that similar full-scale dynamic experiments within the CFF can be performed successfully. Experimentally derived options and considerations are offered in the recommendation section.

- Depending on the options selected and implemented, the personal sampling and surface swiping collective information will be utilized to put into place necessary administrative and engineering controls that will be addressed in the protocol and procedures for a similar dynamic event in the CFF chamber. These methods will serve to address controlling airborne and surface migration of beryllium particulate and/or vapor contamination immediately post-detonation, during re-entry, film retrieval, and chamber clean-up activities, and as a part of ongoing facility maintenance and operations.

\section{Research Parameters}

The research parameters sought in this experimental effort assisted in achieving the specific requirements to address the established goals. The development and implementation of our post-detonation capture equipment yielded appropriate results to directly compare efficiency and post-detonation effects under the variety of conditions 
presented by the METS 04 - 06 dynamic test series. The successful capture of metal analysis samples and investigative and scientific methods were combined to further the science of beryllium capture, detection, and speciation. This experimental process has truly built upon previous developments and successes as well as presenting the challenges and requirements of future post-detonation science conducted by this METS research team. The following research parameters that were achieved are notable:

- All of the stated parameters of the METS $04-06$ experiments were successfully performed within the existing firing tank on the B812 firing table. The newly modified and updated remote sampling equipment performed as designed and were indicative of the needs to further perfect the equipment to capture sequential, replicate samples in the first seconds post-detonation.

- The beryllium experimental parameters based on WCS dynamic test component locations successfully detonated to achieve what is seen as appropriately scaled test conditions based on the collective sampling information. True scaling can be appropriately determined should the same post-detonation collection tank system be connected to the CFF chamber for full-scale analogous dynamic events. The design for a CFF post-detonation collection tank system has been previously submitted and can be adapted to achieve newly derived experimental parameters.

- The opportunity to evaluate beryllium characteristics relative to low and high pressure parameters was achieved for comparisons within and between dynamic events. In all there were three post-detonation windows isolated for direct comparison. The first window is immediately post-detonation and is an appropriate comparison for outdoor explosive events. The second window is directly subsequent to the first and is indicative of a time frame relating to fugitive emissions from the CFF chamber into the B801A ADHZ and common areas.

- Research needs and parameters for achieving results analogous to outdoor dynamic events from indoor chamber sampling protocol are understood as a result of working through the research parameters of this METS. Our collection and evaluation mechanism can also be modified to include the ability to test different metals and gasses associated with individual shot design parameters.

- Based on experimental lessons learned, this METS research team has developed both the protocol and process to provide essential information for authorization requirements relating to outdoor experiments. 


\section{RECOMMENDATIONS}

1. It is possible to conduct full-scale beryllium-containing experimental tests with similar designs within CFF and B801A. These operational procedures should be strongly considered:

a. The overall particulate size produced within the chamber should be increased. The inclusion of chelating agents within pre-shot CFF cardboard containers with a minimum of 600 gallons of water content, and the specific chelater and its amount, should be evaluated;

b. To reduce the concentration of nano-particulate in the chamber and distributed throughout the B801A distribution systems, in addition to the chelation, an extended time period post-test should be addressed to ensure the maximization of particulate size enhancement is achieved before purging the $\mathrm{CFF}$ chamber;

c. An adaptation of approaches toward applications of the scrubber and HEPA systems during the post-shot sequence should be fully evaluated to ensure an integrated environmental, safety, and health approach is achieved; and

d. It is suggested that an additional METS firing tank test be performed, with one more WCS scaled dynamic explosive beryllium-containing device being created, in order to modify the firing tank as outlined above to evaluate the desired effect of increasing the overall particulate size.

2. The CFF chamber entry phases, including re-entry and film retrieval procedures, will need to be adapted in line with abatement techniques for cleaning the chamber, that will be required for work inside a CFF that will contain an elevated concentration of spherical and highly aerosolizable beryllium particulate.

a. When wet swipes, the newly established LLNL CBDPP protocol, are taken on dirty and/or contaminated surfaces they will invariably collect much more mass. There is approximately an order of magnitude difference between wet and dry swipe results even if gross cleaning reduces surface beryllium levels an order of magnitude. However, after the completion of all post-shot cleaning protocol, there is closer to a 2 to 1 differential from wet to dry surface swipe results using this protocol.

3. It is possible to achieve results analogous to outdoor dynamic events from indoor chamber sampling protocol to achieve results for authorization requirement applications. These future experimental parameters were derived as a result of working through the research parameters of this METS protocol. This collection and evaluation mechanism can also be modified to include the ability to test different metals and gasses associated with individual shot design parameters. The applications for this post-detonation science will be 
quite useful for cooperative and collaborative efforts both within LLNL and across the NNSA and DOE complex.

\section{REFERENCES}

1. Dahl, D.A., Johnson, L.J. (1977), “Aerosolized uranium and beryllium from LASL dynamic experiments.” LA-UR-77-681.

2. Shinn, J. (1989), "Beryllium dispersion near explosive firing tables." UCID 21682.

3. Baskett, R. (1991), "Measurements and dispersion of beryllium from high explosive tests at Site 300." Currently in draft form. UCRL-TM-229101.

4. Zalk, D.M. (2002), "METS 01 - 03; Beryllium Sampling." Presentation at LANL - LLNL Hydro Techniques Information Exchange, January 2002, UCRL-PRES221450.

5. Zalk, D. M. (2001), “Metal Analysis Results for SQTS 1 - 5” ES\&H 1-01-056, UCRL-TR-221448.

6. Zalk, D.M., Lyle, J., Arganbright, R., Simmons, L. (2003), "Metals research relating to experimental tests at CFF and Site 300". Internal LLNL presentation September 2003, UCRL-PRES-221447.

7. Zalk, D.M., Ingram, C., Simmons, L., Arganbright R., Koester, C., Lyle, J. (2004). "Material Evaluation Test Series (METS) 07, 08A, and 09A.” UCRL Pending.

8. Kriess, K., Mroz, B., Zhen, H., Wiedmann, Barna, B., (1997), "Risks of beryllium disease related to work processes at a metal, alloy, and oxide production plant. ", Occup. Environ. Med., 54, 605.

9. Eisenbud, M. (1998), "The standard for controlling chronic beryllium disease. “, Appl. Occup. Environ. Hyg. 13, 25

10. Stefaniak, A.B., Hoover, M.D., Day, G.A., Dickerson, R.M., Peterson, E.J., Kent, M.S., Schuler, C.R., Breysse, P.N., Scripsick, R.C. (2004). "Characterization of physicochemical properties of beryllium aerosols associated with prevalence of chronic beryllium disease." J. Environ. Monit., 6, 523

11. Tinkle, S.S., Antonini, J.M., Rich, B.A., Roberts, J.R., Salmen, R., DePree, K., Adkins, E.J., (2003), "Skin as a route of exposure and sensitization in chronic beryllium disease “, Environ. Health Perspect., 111, 1202.

12. Zalk, D.M., Simmons, L., Lyle, J., Ingram, C., Wong, K., Arganbright, R. (2005). Internal LLNL presentation April 2005, UCRL-PRES-221449.

13. ACGIH Threshold Limit Values for Chemical Substances and Physical Agents (2005). ACGIH Worldwide Signature Publications, ISBN; 1-882417-58-5.

14. Martin, J.M., Zalk, D.M., (1998); Comparison of total dust/inhalable dust sampling methods for the evaluation of airborne wood dust. Applied Occupational and Environmental Hygiene. 13, 177 